\title{
Induction of Cytochrome P450 Involved in the Accelerated Blood Clearance Phenomenon Induced by PEGylated Liposomes In Vivo ${ }^{\text {S }}$
}

\author{
Fengling Wang, Yifan Wu, Jiwen Zhang, Huihui Wang, Xiaoting Xie, Xi Ye, Daiyin Peng, \\ and Weidong Chen
}

Institute of Drug Metabolism, School of Pharmaceutical Sciences, Anhui University of Chinese Medicine, Hefei, Anhui, China (F.W., Y.W., H.W., X.X., X.Y., D.P., W.C.); Department of Pharmacy, The Second People's Hospital of Hefei, Hefei, Anhui, China (F.W., X.Y.); and Center for Drug Delivery Systems, Shanghai Institute of Materia Medica, Chinese Academy of Sciences, Shanghai,

China (J.Z.)

Received November 3, 2018; accepted January 16, 2019

\section{ABSTRACT}

Polyethylene glycol (PEG) is recognized as an attractive excipient to modify liposomes due to its extended-circulation properties. Nevertheless, intravenous injection of polyethylene glycol-coated liposomes (PEG-L) usually triggers a rapid systemic clearance of the subsequent dose from blood circulation, which is referred to as an accelerated blood clearance (ABC) phenomenon. Therefore, since the induction of cytochrome P450 (P450) activity may lead to enhanced drug clearance, it motivated us to investigate the possibility of P450 involvement in the ABC phenomenon. In this study, polyethylene glycol-coated liposomal docetaxel was prepared and used to evaluate the magnitude of the $A B C$ phenomenon in rats induced by repeated injection of PEG-modified liposomes. Notably, the $A B C$ phenomenon was observed when the time interval between two doses was from 1 to 7 days, and its magnitude reached the maximum level at 3 days before gradually decreasing the time. Meanwhile, increased activity of CYP3A1, CYP2C6, and CYP1A2 was detected when PEG-L was repeatedly injected in male rats at a 3-day interval. Consistently, the expression levels of hepatic CYP3A1, CYP2C6, and CYP1A2 were also significantly increased in the repeated injection groups and their levels were highest in the 3-day interval group. P450 selective inhibitors confirmed the inhibition of hepatic CYP3A1 was accompanied by an attenuated magnitude of the ABC phenomenon, which strongly suggests that $\mathrm{P} 450 \mathrm{~s}$ may be induced by repeated injection of PEG-L, thus favoring metabolic clearance of the second dose. Collectively, herein, for the first time we demonstrate that the contribution of $\mathrm{P} 450$ s should not be ignored in the ABC phenomenon.
Introduction

Polyethylene glycol (PEG) is now well-recognized as a vital stealth polymer in the drug delivery field, due to its safety, long circulation, and nonimmunogenicity in humans (Suk et al., 2016). Therefore, polyethylene glycol-coated (PEGylated) liposomes (PEG-Ls) are commonly used as drug delivery vehicles to enhance the therapeutic index of entrapped agents, especially for anticancer drugs such as Doxil (Gabizon et al., 2003; Laginha et al., 2005).

Nevertheless, despite the usefulness and excellence of PEGylation, recent reports emphasized that intravenous injection of PEG-L

This work was supported by the National Natural Science Foundation of China [Grant 81773988] and the Natural Science Foundation of Anhui University of Chinese Medicine [Grant 2018zrzd04].

F.W. and Y.W. contributed equally to this work.

https://doi.org/10.1124/dmd.118.085340.

S This article has supplemental material available at dmd.aspetjournals.org. can lead to dramatically reduced half-life $\left(t_{1 / 2}\right)$ of the second dose of PEG-L and accumulation in liver and spleen when repeatedly injected in vivo. This phenomenon is referred to as the accelerated blood clearance (ABC) phenomenon (Dams et al., 2000; Ishida et al., 2005, 2008). Therefore, the ABC phenomenon is a major issue in the clinical application of PEG-L, which requires multiple injections since it limits its passive accumulation in many disease sites. The exact mechanism underlying the $\mathrm{ABC}$ phenomenon remains unclear.

It is proposed that anti-PEG $\operatorname{IgM}$ is produced by spleen cells in response to the first injected PEG-L, which selectively binds to the subsequent dose of PEG-L surface, causing the ABC phenomenon (Ishida et al., 2006b,c; Shimizu et al., 2015). The magnitude of this phenomenon is influenced by the liposomal size, lipid dose, time interval between two injections, drug encapsulation, and animal species (Koide et al., 2012; Yang et al., 2013; Suzuki et al., 2014). Interestingly, the rapid clearance phenomenon has never been reported

ABBREVIATIONS: $\mathrm{ABC}$, accelerated blood clearance; $\mathrm{AUC}_{\mathrm{C} / \mathrm{T}}$, ratio of the area under the concentration-time curve of the control group to the test group; $\mathrm{AUC}_{0-\mathrm{t}}$, area under the plasma concentration-time curve from time 0 to the last quantifiable time point; Chol, cholesterol; $\mathrm{CIM}$, cimetidine; $\mathrm{CL}_{\mathrm{z}}$, plasma clearance; CMC-Na, sodium carboxymethyl cellulose; DSC, differential scanning calorimetry; DTX, docetaxel; EE, encapsulation efficiency; FDA, Food and Drug Administration; HPLC, high-performance liquid chromatography; IS, internal standard; KTZ, ketoconazole; LC-MS/ MS, liquid chromatography-tandem mass spectrometry; MRM, multiple reactions monitoring; MRT $_{0-t}$, mean residence time from time 0 to the last quantifiable time point; P450, cytochrome P450; PEG, polyethylene glycol; PEG-B-L, blank polyethylene glycol-coated liposomes; PEG-DSPE, distearoyl phosphoethanolamine/polyethylene glycol 2000 ; PEG-DTX-L, polyethylene glycol-coated liposomal docetaxel; PEG-L, polyethylene glycol-coated liposomes; PEGylated, polyethylene glycol-coated; $T_{m}$, melting temperature; $t_{1 / 2}$, half-life; $t_{1 / 2-c / r}$, ratio of half-life of the control group to the test group. 
for Doxil/Caelyx and PEGylated liposomal doxorubicin (Ishida et al., 2006a; Cui et al., 2008). It is assumed that these kinds of liposomes with a high cytotoxic drug might be partly taken up by the spleen, and the drug released from the liposomes inhibits the proliferation of splenic marginal zone $\mathrm{B}$ cells and subsequently reduces the secretion of PEG-specific IgM, resulting in failure to induce the ABC phenomenon (Ishida et al., 2006c; Koide et al., 2010). Inconsistently, PEGylated liposomal cytotoxic drugs, such as PEGylated liposomal topotecan and epirubicin, could still elicit a strong ABC phenomenon (Li et al., 2012, 2013; Ma et al., 2012). The contradictory results, therefore, encouraged us to do more detailed research into the ABC phenomenon.

Cytochrome $\mathrm{P} 450$ ( $\mathrm{P} 450)$ proteins are the major phase I metabolic enzymes involved in oxidative biotransformation of drugs and xenobiotics, and most P450s predominantly express in the liver (Guengerich, 2008; Burkina et al., 2017). Consequently, the dramatic increase/induction of P450 activity may lead to enhanced clearance and attenuated pharmacological effects as a result of decreased drug plasma levels (Tracy et al., 2016). It is particularly interesting to understand if $\mathrm{P} 450 \mathrm{~s}$ are involved in the $\mathrm{ABC}$ phenomenon. Seven important P450 isozymes in rats were evaluated in this study: CYP1A2, CYP2C6 (homologous to human CYP2C9), CYP2B1 (homologous to human CYP2B6), CYP2C7 (homologous to human CYP2C8), CYP2D2 (homologous to human CYP2D6), CYP2C11 (homologous to human CYP2C19), and CYP3A1/2 (homologous to human CYP3A4/5), which account for a high percentage $(\sim 70 \%)$ of total $\mathrm{P} 450$ expression in the liver (Lewis et al., 1998; Lin and Lu, 1998; Lu and Li, 2001; IngelmanSundberg, 2004). Probe drugs that are mainly or exclusively metabolized by an individual P450 enzyme can be used to determine the activity of individual P450s in vivo. Recently, relative P450 activity has been determined by quantitative analysis of substrates in a multiple-probe cocktail assay, providing information on the activity of multiple P450 isoforms in a single experiment (Wang et al., 2013; Lee et al., 2015). According to the enzyme selectivity and specificity of probe substrates and criteria for selecting index substrates recommended by the Food and Drug Administration [(FDA); https://www.fda.gov/downloads/drugs/ guidancecomplianceregulatoryinformation/guidances/ucm292362.pdf], we developed a P450 activity cocktail assay using model substrates for the seven important P450s including CYP1A2 (phenacetin), CYP2B1 (bupropion) (Nishiya et al., 2009), CYP2C7 (amodiaquine), CYP2C11 (omeprazole), CYP2D2 (dextromethorphan) (Walsky and Obach, 2004), CYP2C6 (diclofenac), and CYP3A1/2 (midazolam) (Turpeinen et al., 2009).

Docetaxel (DTX), a semisynthetic taxane extracted from the needles of the European yew tree (Taxus baccata) and used in anticancer treatment (Uoto et al., 1997), acted as a model drug for preparing PEGylated liposomal docetaxel (PEG-DTX-L). Notably, $\mathrm{ABC}$ phenomenon was caused by the repeated PEG-L in our pilot experiments. It is very interesting to note that the accumulation of DTX in liver was opposite to that in spleen; the greater the magnitude of the ABC phenomenon, the less accumulation of DTX in liver. In this study, we attempted to investigate the effects of the dose of a high polymer [distearoyl phosphoethanolamine-PEG 2000 (PEG-DSPE)] and the presence of DTX in the first injection as well as various time intervals between two injections on the ABC phenomenon. Furthermore, we evaluated whether the induction of $\mathrm{P} 450$ s is involved in the ABC phenomenon based on the $\mathrm{P} 450$ probe cocktail and pharmacokinetics approaches for the evaluation of enzyme activity. In addition, experiments on the P450 mRNA and protein levels were carried out. Finally, specific P450 inhibitors were employed to verify the contribution of P450s in the systemic clearance of the second PEG-L injection.

\section{Materials and Methods}

\section{Materials}

DTX (purity $>99.0 \%$ ), paclitaxel [internal standard (IS), purity $>99.0 \%$ ], purified egg yolk lecithin, and cholesteryl hemisuccinate were purchased from Ponsure Biotechnology Co., Ltd. (Shanghai, China). PEG-DSPE was obtained from Shanghai Advanced Vehicle Technology Pharmaceutical Ltd. (Shanghai, China). Analytical grade cholesterol (Chol) and sodium carboxymethyl cellulose (CMC-Na) were purchased from Sinopharm Chemical Reagent Co., Ltd. (Shanghai, China). All lipids were used without further purification. Highperformance liquid chromatography (HPLC) grade and liquid chromatographymass spectrometry grade acetonitrile and methanol were obtained from Merck (Darmstadt, Germany). Liquid chromatography-mass spectrometry grade ammonium formate and formic acid were purchased from Fluka Analytical (St. Louis, MO). Docetaxel injection $(0.5 \mathrm{ml}: 20 \mathrm{mg})$ was purchased from Jiangsu Heng Rui pharmaceutical Co., Ltd. (Jiangsu, China). High-purity (>98.0\%) ketoconazole (KTZ), omeprazole, bupropion hydrochloride, diclofenac sodium, dextromethorphan hydrobromide, amodiaquine hydrochloride, phenacetin, and glibenclamide were obtained from the National Institutes for Food and Drug Control (Beijing, China). Cimetidine (CIM) injection $(0.2 \mathrm{~g} / 2 \mathrm{ml})$ was obtained from Shangdong Fangming Pharmaceutical Group Co., Ltd. (Shangdong, China). The forward and reverse primers of all target genes were purchased from Sangon Biotech Co., Ltd. (Shanghai, China). TRIzol reagent (Life Technologies Inc., Carlsbad, CA), RevertAid First Strand cDNA Synthesis Kit (Thermo Fisher Scientific, Vilnius, Lithuania), QuantiNova SYBR Green PCR Kit (QIAGEN, Frankfurt, Germany), Pall nitrocellulose membrane, $0.22 \mu \mathrm{m}$ filter (Solarbio Life Sciences, Ltd., Beijing, China), and an enhanced chemiluminescence reagent kit (Thermo Fisher Scientific, Rockford, IL) were used to detect mRNA and protein. The antibodies used in this work were mouse anti-CYP3A1, anti-CYP2C6, antiCYP1A2, anti-glyceraldehyde-3-phosphate dehydrogenase (Abcam, Cambridge, UK), and anti-mouse horseradish peroxidase-conjugated secondary antibodies (ZSJQ Biotechnology Co., Ltd., Beijing, China). Deionized water was provided by a Milli-Q Reference Ultrapure Water System (Merck). All other reagents were analytical grade.

\section{Preparation of PEG-DTX-L}

The optimal formulation of PEG-DTX-L was determined by a single factor analysis and orthogonal test (Gai et al., 2018). PEG-DTX-L, composed of DTX: Chol:Chol hemisuccinate:PEG-DSPE (lipids):lecithin at molar ratios of 1.0:7.5: 6.6:4.6:25.5, was prepared by the ethanol injection method as described previously with minor modifications (He et al., 2017). In brief, the lipids and DTX were dissolved in $3 \mathrm{ml}$ absolute ethanol, followed by heating to $50^{\circ} \mathrm{C}$. The mixtures were then rapidly injected into $20 \mathrm{ml}$ of stirred $(800 \mathrm{rpm}) \mathrm{PBS}(\mathrm{pH}=$ 7.4). Then, suspensions were kept at $50^{\circ} \mathrm{C}$ under stirring for 2 hours using an electric stirrer (DF-1 Electric Stirrer, Youlian Instruments, Jintan, China). The obtained multilamellar vesicles were passed through a high-speed homogenizer (ANGNI AD200L-H; Angni Instruments, Shanghai, China) for 15 minutes at $10,000 \mathrm{rpm}$ to harvest the small unilamellar PEG-DTX-L. Blank PEGylated liposomes (PEG-B-L) were prepared according to the same procedure as for PEGDTX-L without adding DTX.

Drug content was assayed in triplicate using a validated HPLC method (Waters 1525; Waters) as reported previously (Huang et al., 2013). The HPLC system was equipped with a Waters 1525 binary HPLC pump and Waters 2489 UV detector. The stationary phase was performed on a COSMOSIL $\mathrm{C}_{18}$ reversed-phase column $\left(4.6 \times 250 \mathrm{~mm}, 5 \mu \mathrm{m}\right.$; Nacalai Inc., Kyoto, Japan) at $30^{\circ} \mathrm{C}$. The mobile phase consisted of acetonitrile: $0.1 \%$ phosphoric acid solution $(55: 45, \mathrm{v} / \mathrm{v})$ with a flow rate of $1.0 \mathrm{ml} / \mathrm{min}$. The UV detection for DTX was performed at $230 \mathrm{~nm}$. The injection volume was $20 \mu \mathrm{l}$ and the retention time for DTX was about at 8.60 minutes. The calibration curve was linear at a range of $0.05-10 \mu \mathrm{g} / \mathrm{ml}$ with the corresponding linear regression equation of $Y=29,518 X-835.02$ and a correlation coefficient of $r=0.9993$.

\section{Characterization of PEG-L}

Morphologic Examination. The morphology of the liposomes was carried out using a transmission electron microscope (TEM HT7700; JEOL Ltd., Japan). Before observation, the liposome samples were analyzed after being diluted five times with deionized water to a count rate of $100-300 \mathrm{kcps}$, and then a drop of 
PEG-L was plated on a coverslip for about 5 minutes. After being dried at room temperature, the liposomes were observed by transmission electron microscopy.

Particle Size and Zeta Potential Analysis. The particle size, polydispersity index, and zeta potential of the PEG-L were measured by dynamic light scattering, using a Zetasizer size analyzer (Nano ZS90; Malvern Instruments, Malvern, UK) at $25^{\circ} \mathrm{C}$. The instrument was calibrated with standard latex liposomes. All measurements of particle size were performed after the liposomes were diluted 20-fold in deionized water. Measurement of the zeta potential for the prepared liposomes was performed without dilution. Experimental values are presented from three different results.

Determination of the Entrapment Efficiency. The DTX encapsulated in the PEG-L was measured indirectly by the HPLC method as described previously (He et al., 2017). In brief, the free DTX (nonencapsulated) was separated by an ultrafiltration/centrifugation technique using an Amicon Ultra-4 ultrafiltration centrifuge tube (the molecular weight cutoff was $100 \mathrm{~K}$; Millipore Corporation). The liposomes were properly diluted with deionized water and centrifuged at $3000 \mathrm{rpm}$ for 30 minutes. The filtrate was obtained and determined by HPLC as free DTX. Drug-loaded liposomes were mixed with methanol for liposome disruption and dissolved in the mobile phase, which was subjected to ultrasound (KQ 300B; Kunshan ultrasonic Instruments Co., Ltd., China) for 5 minutes to make the drug completely free of liposomes. Then, the total DTX in the liposomes was determined by HPLC after the solution was filtered through a $0.22 \mu \mathrm{m}$ syringe filter. The drug encapsulation efficiency (EE) was calculated based on the following equation: $\mathrm{EE}(\%)=\left[\left(\mathrm{W}_{\text {total }}-\mathrm{W}_{\text {free }}\right) / \mathrm{W}_{\text {total }}\right] \times 100 \%$, where $\mathrm{W}_{\text {total }}$ and $\mathrm{W}_{\text {free }}$ are the weight of the total drug in the system and the weight of the free drug in the filtrate, respectively. Each experiment was independently conducted three times.

Differential Scanning Calorimetry. Differential scanning calorimetry (DSC) experiments were employed to investigate the change in the PEG-L crystalline structure using a DSC Q2000 calorimeter (TA Instruments). A hermetically sealed aluminum pan was filled with approximately $5 \mathrm{mg}$ of the sample, while an empty pan was used as the reference. The data for DTX, Chol, Chol hemisuccinate, PEGDSPE, lecithin, their physical mixture, lyophilized PEG-DTX-L, and PEG-B-L were recorded at a scan rate of $10^{\circ} \mathrm{C} / \mathrm{min}$ from 20 to $200^{\circ} \mathrm{C}$.

In Vitro Release Kinetics. The dialysis bag diffusion technique was used to study the in vitro drug release of DTX from the PEG-L according to a procedure similar to that described by $\mathrm{He}$ et al. (2017). Briefly, PEG-L dispersion equivalent to $0.5 \mathrm{mg}$ of drug was filled into a dialysis membrane bag (21 mm, MVCO800014400 Da, Milipore.), hermetically sealed and immersed into a beaker containing $100 \mathrm{ml}$ of PBS (pH 7.4) and $0.1 \% \mathrm{w} / \mathrm{v}$ Tween-80. The entire system was kept at $37 \pm 0.5^{\circ} \mathrm{C}$ with continuous stirring at $100 \mathrm{rpm}$. Samples were taken out from the beaker at predetermined time intervals and replaced with fresh medium to maintain the sink condition. The amount of DTX released from PEG-L was analyzed by HPLC as described previously (to determine the entrapment efficiency). The cumulative percentage release was then calculated from the amount of drug release. Each releasing experiment was carried out in triplicate.

\section{Experimental Animals and Ethics Statement}

Male Sprague-Dawley rats ( 5 weeks old; $220 \pm 15 \mathrm{~g}$ ) were obtained from the Experimental Animal Center of Anhui Medical University (Hefei, China). The experimental animals were allowed free access to water and a commercial rodent diet. They were adapted to the controlled experimental conditions of $25 \pm 2^{\circ} \mathrm{C}$, humidity $60 \% \pm 5 \%$, with a 12-hour light/dark cycle for 1 week before experiments. All procedures involving experimental animals were carried out in accordance with the guidelines evaluated and approved by the Institutional Animal Care and Use Committee, Anhui University of Chinese Medicine.

\section{Pharmacokinetics and Biodistribution of PEG-L after Repeated Administration}

Injection Protocols. Male Sprague-Dawley rats were randomly and equally divided into five groups for pharmacokinetics and biodistribution investigations according to a completely randomized digital table, and formulations were injected intravenously via tail vein ( $n=6$ for each group). PEG-DTX-L and PEGB-L were freshly prepared before administration. For the purpose of comparison of the effect of different time intervals on the magnitude of the $\mathrm{ABC}$ phenomenon, PEG-DTX-L was injected into animals after the first injection of PEG-B-L for $1,3,5$, or 7 days. Rats in the $1,3,5$, and 7 days groups all received two doses, PEG-B-L (containing $0.05 \mu \mathrm{mol}$ of PEG-DSPE $/ \mathrm{kg}$ ) on day 0 and then $2.5 \mathrm{mg} / \mathrm{kg}$ PEG-DTX-L after $1,3,5$, or 7 days. Rats in the 0 day group were only treated with a single injection of $2.50 \mathrm{mg} / \mathrm{kg}$ PEG-DTX-L, which was used as a control. Here, the dose of PEG-DTX-L represented the amount of drug that was not phospholipid. The detailed injection protocols for PEG-DTX-L and PEG-B-L are presented in Table 1.

Sample Preparation. About $0.3 \mathrm{ml}$ orbital venous blood samples were collected under isoflurane anesthesia into sodium heparin tubes immediately prior to (blank sample) and following the predetermined postinjection time points $(0.167,0.5,1,2,4,6,8,10$, and 12 hours). Plasma samples were centrifuged at $3000 \mathrm{rpm}$ for 10 minutes, and subsequently plasma $(100 \mu \mathrm{l})$ was obtained and stored at $-80^{\circ} \mathrm{C}$ until further liquid chromatography-tandem mass spectrometry (LC-MS/MS) analysis.

Rats were euthanized with isoflurane anesthesia after the last blood sample was harvested at 12 hours, and tissues of interest (liver and spleen) were collected and flash frozen in liquid nitrogen and stored at $-80^{\circ} \mathrm{C}$. Upon processing, tissues were thawed and diluted in a 1:2 ratio (w/v) with PBS ( $\mathrm{pH} 7.4)$ prior to homogenizing with a T18 digital ULTRA-TURRAX homogenizer (IKA Works Guangzhou, Guangzhou, China). Plasma or tissue homogenate was further processed by 3 -fold of methyl tert-butyl ether with $250 \mathrm{ng} / \mathrm{ml}$ paclitaxel (IS) and swirled for 5 minutes. After centrifuged at $12,000 \mathrm{rpm}$ for 5 minutes at $4^{\circ} \mathrm{C}$, the supernatant was removed to a clean tube, evaporated to dryness under nitrogen, and reconstituted in $100 \mu \mathrm{l}$ of methanol. The samples were then swirled, transferred to autosampler vials, and analyzed by LC-MS/MS.

LC-MS/MS Conditions. The chromatographic system consisted of a G6460H MS/MS spectrometer (Agilent Technologies Inc., Waldbronn, Germany) coupled to an Agilent 1260 series HPLC system (Agilent Technologies Inc., Shanghai, China). Chromatographic separations were carried out using a DIKMA Leapsil $\mathrm{C}_{18}$ column $(3.0 \times 50 \mathrm{~mm}, 2.7 \mu \mathrm{m})$, maintained at $30^{\circ} \mathrm{C}$.

The liquid chromatography mobile phase consisted of water containing $1 \mathrm{mM}$ ammonium formate, acidified to $\mathrm{pH} 6.5$ with formic acid (solvent $\mathrm{A}$ ) and methanol (solvent B) in a 27:73 ratio (v/v) with a flow rate of $0.4 \mathrm{ml} / \mathrm{min}$ and an injection volume of $5 \mu \mathrm{l}$. The overall run time was 4.0 minutes. The mass spectrometer was operated in the positive electrospray ionization mode. The detection was operated in the multiple reactions monitoring (MRM) mode. The optimized MRM transitions were mass-to-charge $830.4 \rightarrow 549.3$ with a fragment voltage of $135 \mathrm{~V}$ and a collision energy of $15 \mathrm{~V}$ for DTX and mass-to-charge

TABLE 1

Injection protocols for PEG-B-L and PEG-DTX-L

Day 0 indicates the group received only a single injection of PEG-DTX-L. Days 1, 3, 5, and 7 indicate these groups received two injections of PEG-L at different time intervals $(1,3,5$, and 7 days, respectively).

\begin{tabular}{lccc}
\hline Group & First Injection Liposomes & Time Interval & Second Injection $(2.5 \mathrm{mg} / \mathrm{kg})$ \\
\hline day & PEG-DTX-L $(2.50 \mathrm{mg} / \mathrm{kg}$, containing & day & None \\
0 & $0.05 \mu \mathrm{mol}$ of PEG-DSPE/kg) & 0 & PEG-DTX-L \\
& PEG-B-L $(0.05 \mu \mathrm{mol}$ of PEG-DSPE $/ \mathrm{kg})$ & 1 & PEG-DTX-L \\
3 & PEG-B-L $(0.05 \mu \mathrm{mol}$ of PEG-DSPE $/ \mathrm{kg})$ & 3 & PEG-DTX-L \\
5 & PEG-B-L $(0.05 \mu \mathrm{mol}$ of PEG-DSPE $/ \mathrm{kg})$ & 5 & PEG-DTX-L \\
7 & PEG-B-L $(0.05 \mu \mathrm{mol}$ of PEG-DSPE $/ \mathrm{kg})$ & 7 & \\
\hline
\end{tabular}


$876.8 \rightarrow 308.5$ with a fragment voltage of $115 \mathrm{~V}$ and a collision energy of $18 \mathrm{~V}$ for paclitaxel (IS), respectively. Representative parent ion and product ion spectrum of DTX and IS are given in Supplemental Fig. 1. Typical chromatograms of DTX and IS showed that no interfering peak in the blank matrices was observed under these experimental conditions (Supplemental Fig. 2).

Method Validation. The LC-MS/MS methods for analysis of plasma and tissue samples were linear in the concentration ranges of $2.5-2000 \mathrm{ng} / \mathrm{ml}$ $(r=0.999)$ and $5-1000 \mathrm{ng} / \mathrm{ml}(r=0.995)$, respectively. Calibration curves were established by employing a linear regression with a weighting factor of $1 / x^{2}$. The lowest limits of quantification for plasma and tissue samples estimated at a signalto-noise ratio near or above 10 were 2.5 and $5 \mathrm{ng} / \mathrm{ml}$, respectively. For intra- and interassay accuracy, performance was assessed by analyzing five replicates of samples at four levels of quality control concentrations (lowest limit of quantification, low quality control, middle quality control, and high quality control) on three consecutive days. The median recoveries for intra- and interassay of DTX ranged from $98.4 \%$ to $107.6 \%$ in plasma, $99.6 \%-104.8 \%$ in liver, and 99.9\%-113.6\% in spleen. The intra- and interassay CV values of DTX in the plasma, liver, and spleen samples ranged from $5.2 \%$ to $17.5 \%, 4.4 \%$ to $17.9 \%$, and $3.7 \%$ to $14.2 \%$, respectively (Supplemental Table 2). The stabilities of DTX in plasma, liver, and spleen were evaluated at the low and high quality control levels with six replicates under different conditions, including stock solution, autosampler, short-term, long-term, and freeze-thaw stability (Supplemental Table 3). The results demonstrated five types of stability were within the acceptance criteria. Six different lots of blank plasma were used to assess the extraction efficiencies and matrix effects at three quality control levels. The extraction recovery of DTX, as determined in six replicates, was about $78.6 \%$ $89.4 \%$ in plasma, $82.0 \%-96.8 \%$ in liver, and $90.3 \%-99.1 \%$ in spleen. The CVs for the three levels were all less than $10 \%$. The IS-normalized matrix factor was recommended as a marker to evaluate the matrix effect (Bansal and DeStefano, 2007). The matrix factors of DTX in plasma and tissue varied from $92.7 \%$ to $107.9 \%$ with CV values less than $15.0 \%$ (Supplemental Table 4). No significant interference in the blank matrices was observed from other endogenous substances in this method, indicating feasible sample preparation. These results all met the acceptance criteria of the FDA guideline for bioanalytical method validation (https://www.fda.gov/downloads/drugs/guidancecomplianceregulatoryinformation/guidances/ucm070107.pdf), suggesting that the developed method could be applied for the pharmacokinetic study of DTX. A noncompartmental model was used to calculate the pharmacokinetic parameters of different animal groups after repeated injection of PEG-L using the DAS 2.0 software program (Shanghai Bojia Medical Technology Co., Ltd., Shanghai, China).

\section{Determination of P450 Activity by LC-MS/MS}

Experimental Design. Male Sprague-Dawley rats were randomly divided into five groups ( $n=8$ per group). Both PEG-L and P450 probe cocktail solution were freshly prepared before administration. For the purpose of comparing the effect of single and repeated injections of PEG-L (with a 3-day interval) on P450 activity, all animals received intravenous injection of the probe cocktail solution containing phenacetin $(0.4 \mathrm{mg} / \mathrm{kg})$, bupropion $(0.4 \mathrm{mg} / \mathrm{kg})$, amodiaquine $(1.2 \mathrm{mg} / \mathrm{kg})$, omeprazole $(0.4 \mathrm{mg} / \mathrm{kg})$, dextromethorphan $(0.4 \mathrm{mg} / \mathrm{kg})$, diclofenac $(0.4 \mathrm{mg} / \mathrm{kg})$, and midazolam $(0.4 \mathrm{mg} / \mathrm{kg})$ dissolved in physiologic saline (containing 3\% absolute ethanol) after single or repeated PEG-L injection for 30 minutes, which was used in the single and repeated PEG-L injection groups. To exclude the influence of DTX itself (in the burst release of DTX at the initial time from the surface or encapsulated in the outermost layers), the same dose of DTX injection was injected into animals with a single injection or repeated injections (with a 3-day interval) before the treatment with the probe cocktail solution, which was used in the single and repeated DTX injection groups. The detailed PEG-L/P450 probe-drug administration is described in Table 2. Control rats were injected with the same volume of PBS as the two doses.

Blood Sample Preparation. Blood samples $(0.3 \mathrm{ml})$ were collected from the orbital venous under isoflurane anesthesia into heparinized $(1.5 \mathrm{ml})$ polythene tubes immediately prior to (blank sample) and following the predetermined time points $(3,10,30,45,60,90,120,240$, and 360 minutes) after injection of probe cocktail solution. Plasma samples were immediately centrifuged at $3000 \mathrm{rpm}$ for 10 minutes. Subsequently, plasma $(100 \mu \mathrm{l})$ was obtained and stored at $-80^{\circ} \mathrm{C}$ until further LC-MS/MS analysis.

LC-MS/MS Conditions. The chromatographic system is the same as previously mentioned. The liquid chromatography mobile phase consisted of water containing $0.1 \%$ formic acid (solvent A) and acetonitrile (solvent B). The binary solvent gradient elution $(0.2 \mathrm{ml} / \mathrm{min}$ flow rate) was optimized as follows: $10 \% \mathrm{~B}$ at the time of injection, a linear increase to $80 \% \mathrm{~B}$ for 1 minute, remaining at $80 \%$ B for 1.3 minutes, an increase to $95 \%$ B for 2 minutes, a decrease to $90 \%$ B for 3 minutes, remaining at $90 \%$ B for 3.5 minutes, decrease to $10 \%$ B for 5 minutes, and maintained at $10 \% \mathrm{~B}$ for an additional 3 minutes for re-equilibration.

Quantitation was performed by MRM in the positive electrospray ionization mode and the related product ion for each probe drug. The optimized MRM transitions, fragment voltages, and collision energies determined for each probe drug and IS are listed in Supplemental Table 7. The typical chromatographs (Supplemental Fig. 3) indicate that the response of endogenous compounds had no significant influence on determining the probe drugs under the current conditions.

Method Validation. To evaluate the sensitivity, reliability, and specificity of the LC-MS/MS method, the lowest limit of quantification, linearity of standard curve, accuracy, precision stability, extraction efficiency, and matrix effect for each probe drug were validated. As shown in Supplemental Fig. 3 and Supplemental Tables 9-11, all of results for selectivity, sensitivity, linearity, accuracy, precision, stability, extraction efficiency, as well as matrix effect met the requirement in the FDA bioanalytical method validation guidance (the detailed results are described in the Supplemental Material), indicating that the developed method could be applied to determine the levels of all probe drugs in rat plasma. The DAS 2.0 software program was used to estimate the pharmacokinetic parameters calculated by the noncompartmental model, and the parameters of the seven substrates were used to represent the relative activity of the seven isozymes.

\section{Real-Time Quantitative Polymerase Chain Reaction}

Total RNA isolation and cDNA synthesis as well as real-time quantitative polymerase chain reaction were performed as described previously (Rio et al., 2010; Doak and Zaï, 2012; Roth et al., 2018). Briefly, total RNA was extracted from tissue samples using TRIzol reagent following the manufacturer's protocol. RNA concentration and purification were determined spectrophotometrically using a SoftMax Pro 5 Multiscan Spectrum (Molecular Devices, Silicon Valley, $\mathrm{CA})$ with the $\mathrm{OD}_{260} \mathrm{~nm} / \mathrm{OD}_{280} \mathrm{~nm}$ ratio expected to be $1.8-2.0$. Total RNA $(5 \mu \mathrm{g})$ extracted from each tissue was reverse transcribed using the RevertAid First Strand cDNA Synthesis Kit, according to the manufacturer's instructions. Subsequently, all cDNA samples were diluted 30 times and stored at $80^{\circ} \mathrm{C}$ until

\section{TABLE 2}

Injection protocols for cocktail probes

Day 0 indicates these groups received only a single injection of DTX injection or PEG-DTX-L. Day 3 indicates these groups received repeated injections of DTX or $P E G$-L with a 3-day interval.

\begin{tabular}{|c|c|c|c|}
\hline Group & First Injection & Time Interval & $\begin{array}{c}\text { Second Injection }(2.5 \mathrm{mg} / \mathrm{kg})+\text { Cocktail } \\
\text { Probes }(1.0 \mathrm{mg} / \mathrm{kg})\end{array}$ \\
\hline & & day & \\
\hline Control & PBS & 3 & PBS + cocktail probes \\
\hline Single DTX injection & DTX injection $(2.5 \mathrm{mg} / \mathrm{kg})$ & 0 & Cocktail probes \\
\hline Repeated DTX injection & DTX injection $(2.5 \mathrm{mg} / \mathrm{kg})$ & 3 & DTX injection + cocktail probes \\
\hline Single PEG-L injection & PEG-DTX-L $(2.5 \mathrm{mg} / \mathrm{kg})$ & 0 & Cocktail probes \\
\hline Repeated PEG-L injection & PEG-B-L & 3 & PEG-DTX-L + cocktail probes \\
\hline
\end{tabular}


use. For the assessment of RNA expression, cDNA was amplified using a StepOne Plus PCR System (Applied Biosystems) with the SYBR Green PCR kit and the forward and reverse primers (details of the primer sequences used are given in Supplemental Table 12). The cycling parameters were performed as follows: one cycle of initial denaturation $\left(95^{\circ} \mathrm{C}, 10\right.$ minutes $)$, followed by quantification with 40 cycles of denaturation $\left(95^{\circ} \mathrm{C}, 15\right.$ seconds) and annealing $\left(60^{\circ} \mathrm{C}, 60\right.$ seconds). Finally, the specificity of the target amplification was confirmed by an additional cycle $\left(95^{\circ} \mathrm{C}\right.$ for 15 seconds, $60^{\circ} \mathrm{C}$ for 60 seconds, and $95^{\circ} \mathrm{C}$ for 15 seconds). For all calculations regarding quantitative polymerase chain reaction, the comparative $\mathrm{CT}^{2-\Delta \Delta \mathrm{CT}}$ method was applied to determine the relative mRNA expression using $\beta$-actin as an endogenous reference gene. Each real-time quantitative polymerase chain reaction was performed in triplicate.

\section{Western Blotting}

For protein analysis, about $50 \mathrm{mg}$ of liver tissue was homogenized and lysed in $1 \mathrm{ml}$ of radioimmunoprecipitation assay lysis buffer for 30 minutes on ice. Total protein concentration was quantified by the bicinchoninic acid protein assay method. Subsequently, the lysates were diluted in $5 \times$ SDS-PAGE loading buffer containing $5 \% \beta$-mercaptoethanol, denatured by boiling 10 minutes at $95^{\circ} \mathrm{C}$, and protein samples $(20 \mu \mathrm{g})$ were separated by $12 \%$ SDS-PAGE. After proteins were transferred to nitrocellulose membrane using wet blotting on ice $(200 \mathrm{~mA}$, 2 hours) and blocked with $5 \%$ skimmed milk in Tris-buffered saline containing $0.05 \%$ Tween-20 at room temperature for 2 hours, the membranes were then incubated with an appropriate dilution of the primary antibody at $4{ }^{\circ} \mathrm{C}$ overnight. After washing with Tween-20, the membranes were incubated in the appropriate horseradish peroxidase-conjugated secondary antibody in blocking solution at room temperature for 1 to 2 hours. Finally, the bands were detected by using the enhanced chemiluminescence detection kit according to the manufacturer's instructions and expression levels were normalized to glyceraldehyde-3-phosphate dehydrogenase. The determination of all protein expression was performed in triplicate and the results were reproducible. Autoradiographs were scanned using the Alpha Chemiluminescent gel imaging system FluorChem FC3 (ProteinSimple, Silicon Valley, CA) and quantified using the ImageJ software (version 1.45S; National Institutes of Health).

\section{Inhibition Study of the ABC Phenomenon In Vivo}

To verify the role of P450 enzymes in the process of PEGylated liposomeinduced $\mathrm{ABC}$ phenomenon, the relative selective $\mathrm{P} 450$ inhibitors were used in this study, including KTZ (a strong inhibitor of CYP3A1 as well as a weak inhibitor of CYP1A2 and CYP2C6 in rats) and CIM (a strong inhibitor of CYP2C6 and a weak inhibitor of CYP1A2 in rats), with reference to the recommended guidance by the FDA (https://www.fda.gov/drugs/developmentapprovalprocess/developmentresources/druginteractionslabeling/ucm093664.htm\#table3-2) and previous reports (Meredith et al., 1985; Mosca et al., 1985; Levine et al., 1998; Kotegawa et al., 2002; Yang et al., 2018). The inhibition study consisted of six groups ( $n=6$ for each group). The rats were administered the following treatments: 1 ) to inhibit CYP3A1, rats in group 1 were pretreated with oral administration of KTZ (suspended in $0.5 \%$ CMC-Na, $100 \mathrm{mg} / \mathrm{kg}$ per day) for 7 days, and treated with the first injection of PEG-B-L on the fourth day, with a repeated injection of PEGDTX-L $(2.5 \mathrm{mg} / \mathrm{kg})$ on the seventh day (3-day KTZ+); 2) rats in group 2 were pretreated with an equal volume of $0.5 \% \mathrm{CMC}-\mathrm{Na}$ for 7 days, and treated with the first injection of PEG-B-L on the fourth day, with a repeated injection of PEGDTX-L (2.5 mg/kg) at the seventh day (3-day $\mathrm{KTZ}-$ ); 3) rats in group 3 were pretreated with an equal volume of $0.5 \% \mathrm{CMC}-\mathrm{Na}$ for 7 days followed by a single injection of $2.5 \mathrm{mg} / \mathrm{kg}$ PEG-DTX-L (control); 4) to inhibit CYP2C6, rats in group 4 received the first injection of $\mathrm{PEG}-\mathrm{B}-\mathrm{L}$ on the first day, and 3 days later rats were pretreated with a single intraperitoneal dose $(150 \mathrm{mg} / \mathrm{kg}$, dissolved in $0.9 \%$ saline $)$ of CIM for 1.5 hours before the second dose of $2.5 \mathrm{mg} / \mathrm{kg}$ PEG-DTX-L (3-day $\mathrm{CIM+)}$; 5) rats in group 5 received the first injection of PEG-B-L on the first day, and 3 days later rats were pretreated with an equal volume of $0.9 \%$ saline for 1.5 hours before the repeated injection of $2.5 \mathrm{mg} / \mathrm{kg}$ PEG-DTX-L (3-day CIM-); and 6) rats in group 6 were pretreated with an equal volume of $0.9 \%$ saline for 1.5 hours followed by a single injection of $2.5 \mathrm{mg} / \mathrm{kg}$ PEG-DTX-L (control). Here, PEG-B-L in all groups was the same (containing $0.05 \mu \mathrm{mol}$ of PEG-DSPE/kg). The preparation and detection conditions in LC-MS/MS for blood and tissue samples were the same as mentioned previously.

\section{Statistical Analysis}

All data subjected to statistical analysis were obtained from at least three parallel experiments. Data are expressed as mean \pm S.D. Each set of results was first evaluated for normal distribution using the Kolmogorov-Smirnov test. Normally distributed data of multiple comparisons were analyzed by one-way ANOVA followed by a Student-Newman-Keuls post-hoc test for statistical comparisons of two groups (IBM SPSS 23.0 software; IBM Corporation, Armonk, NY). In Supplemental Tables 15-17, the differences in pharmacokinetic parameters were examined using a nonparametric test (Kruskal-Wallis) followed by Dunn's test. The levels of significance were set at $P<0.05$ and $P<0.001$. The pharmacokinetic parameters were calculated by noncompartmental model analysis using the DAS 2.0 software package (Shanghai Bojia Medical Technology Co., LTD.).

\section{Results}

\section{Characterization of PEG-L}

Particle Size, Polydispersity, Zeta Potential, EE, and Morphology Analysis. Generally, the physiochemical properties of colloidal systems including the mean particle size, polydispersity index, and zeta potential are strongly associated with their performance and biologic activity. As shown in Supplemental Table 13 and Supplemental Fig. 4, the mean particle sizes of before loading and after loading were similar (about $110 \mathrm{~nm}$ ), owing to the presence of PEG on the surface of the liposomal carrier, resulting in the formation of steric hindrance on the liposome surface (Immordino et al., 2006). Moreover, the formulation had a narrow size distribution, with a polydispersity index value ranging from 0.127 to 0.173 , indicating the PEG-L dispersed uniformly. The analysis of the zeta potential is a critical parameter used to predict the physical storage of delivery systems. A higher absolute value of the zeta potential indicates greater repulsion between particles, and aggregation is less likely to occur. In this study, all liposomes were negatively charged $(-27.0 \pm 0.35 \mathrm{mV}$ for PEG-B-L; $-32.40 \pm 1.04 \mathrm{mV}$ for PEGDTX-L) due to the presence of PEG-DSPE on the surface. As shown in the transmission electron microscopy image (Supplemental Fig. 5), liposomes display uniform diameters in the nanometer scale and disperse homogeneously with a regular spherical shape, which is consistent with the dynamic light scattering results. In addition, this formulation displayed a high EE value of $96.62 \% \pm 0.95 \%$, and no aggregation was found among the particles.

DSC Study. DSC studies were performed to investigate the phase transition temperature of the PEG-DTX-L formulation along with its individual components. Each melting temperature $\left(T_{m}\right)$ is different when comparing the sharp peaks in Supplemental Fig. 6. The DSC thermogram of raw material DTX was a wide peak at $163.33^{\circ} \mathrm{C}$ (curve B), and relatively sharp peaks at $150.04,55.09,125.86$, and $185.75^{\circ} \mathrm{C}$ were obtained from Chol, PEG-DSPE, lecithin, and Chol hemisuccinate, respectively (curves $\mathrm{E}, \mathrm{F}, \mathrm{G}$, and $\mathrm{H}$ ). The $\mathrm{T}_{\mathrm{m}}$ of the physical mixture was near $51.04^{\circ} \mathrm{C}$ (curve D), which was the lowest $\mathrm{T}_{\mathrm{m}}$ in the DSC thermograms. The incorporation of DTX in the liposome formulation had increased the $\mathrm{T}_{\mathrm{m}}$ of PEG-B-L by $3.7^{\circ} \mathrm{C}$ and affected the transition phase behavior as detected by DSC. This confirms that the incorporation of DTX and other materials leads to a change in the liposome crystalline structure.

In Vitro Release Kinetics of PEG-DTX-L. In general, the release rate of drug is important in colloidal delivery systems, which is associated with the in vivo performance. To evaluate the controlled release potential of PEG-DTX-L, release profiles of free DTX and DTX from PEG-L were investigated up to 36 hours (Supplemental Fig. 7). As expected, more than $80 \%$ of free DTX was measured in the initial 2 hours. Obviously, the dissolution and diffusion rate of PEG-DTX-L was much slower than the free DTX solution. Release dosing in the initial 0.5 hour was nearly $30.2 \%$, which may be attributed to the 
adsorbed drug on the surface or encapsulated in the outermost layers of the liposomes, followed by a slow and sustained profile, reaching approximately $46.2 \%$ of released drug after 36 hours. Therefore, the accumulative release profiles demonstrate that the lipid bilayer can change the rate of dissolution and diffusion of DTX under the described experimental conditions. The obtained release kinetic model of free DTX and PEG-DTX-L both fit well with first-order kinetics: $\ln (1-Q)=-0.2401 t-0.9888, r=0.8478$, and $\ln (1-Q)$ $=-0.2702 t-0.7427, r=0.8485$, respectively.

\section{Effect of PEG-L with Time Intervals on the Induction of the ABC Phenomenon upon Repeated Injection}

As revealed by our study (the detailed results are described in the Supplemental Material), the initial injection of PEG-B-L triggered an apparent $\mathrm{ABC}$ phenomenon that significantly accelerated the blood clearance rate of subsequently injected liposomes, which was comparable with that of DTX encapsulated in liposomes (Supplemental Fig. 8; Supplemental Tables 5 and 6), suggesting the liposomes induced the ABC phenomenon and not DTX. The results are consistent with those of previous studies performed for liposomes (Ma et al., 2012), indicating that PEG-L can induce ABC phenomenon by itself after repeated injection, and the addition of DTX in PEG-L did not alter what was seen with the blank formulation. Moreover, the PEG-DSPE content of the liposomes that were initially injected did not affect the extent of the ABC phenomenon within the range of $0.025-0.05 \mu \mathrm{mol} / \mathrm{kg}$ of PEG-B-L in this study (Supplemental Fig. 8; Supplemental Tables 5 and 6), which is in agreement with the results by Ishihara et al. (2009), who reported that the extent of the $\mathrm{ABC}$ phenomenon was not apparently changed with the increased dose of PEG polymer in the initial dose. It was also confirmed that a specific range of PEG polymer predose does not affect $\mathrm{ABC}$ induction, but that a larger proportion of PEG polymer inhibited the ABC phenomenon (Saadati et al., 2013).

The issue of whether the time interval between two injections has an influence on the induction of the enhanced clearance effect was assessed via comparing the pharmacokinetics and biodistribution behavior upon repeated injections. Rats in five groups $(1,3,5$, and 7 days) were injected with two injections of PEG-L at various time intervals $(1,3,5$, and 7 days, respectively) between the initial and second dose. The ABC phenomenon was evaluated by examining the area under the concentration-time curve and $t_{1 / 2}$ of the experimental payload (DTX). The ratio of the area under the concentration-time curve of the control group to the test group $\left(\mathrm{AUC}_{\mathrm{C} / \mathrm{T}}\right)$ and the ratio of $\mathrm{t}_{1 / 2}$ of the control group to the test group $\left(t_{1 / 2-C / T}\right)$ were previously used as major markers to assess the altered pharmacokinetic profiles of these groups ( $\mathrm{Xu}$ et al., 2015). The results shown in Supplemental Table 14 and Fig. 1A are similar to those reported in previous studies (Ishida et al., 2006a; Cui et al., 2008); the pharmacokinetic profiles of the four test groups were dramatically different compared with the single dose control. The ABC phenomenon occurred after repeated injection of PEG-DTX-L at different time intervals $(1,3,5$, and 7 days). The values of the area under the plasma concentration-time curve from time 0 to the last quantifiable time point $\left(\mathrm{AUC}_{0-\mathrm{t}}\right)$ and mean residence time from time 0 to the last quantifiable time point $\left(\mathrm{MRT}_{0-\mathrm{t}}\right)$ in the test groups $(1,3,5$, and 7 days) were significantly decreased compared with control (0 day group). Particularly for the 3 days group, the value of plasma clearance $\left(\mathrm{CL}_{\mathrm{z}}\right)(0.74 \pm 0.16 \mathrm{l} / \mathrm{h}$ per kilogram $)$ was significantly higher $(P<0.01)$ and the values of $\mathrm{AUC}_{0-\mathrm{t}}, \mathrm{MRT}_{0-\mathrm{t}}$, and $\mathrm{t}_{1 / 2}(3053.35 \pm 587.38 \mu \mathrm{g} / \mathrm{l} \cdot \mathrm{h}$, $3.84 \pm 0.31$, and $4.09 \pm 1.05$ hours, respectively) were distinctly lower $(P<0.05)$ than those of the control. Thus, the magnitude of the ABC phenomenon reached the maximum level at 3 days after the initial dose. In the 5 and 7 days groups, the $\mathrm{CL}_{\mathrm{z}}$ value of PEG-DTX-L $(0.50 \pm 0.16$ and $0.33 \pm 0.12 \mathrm{l} / \mathrm{h}$ per kilogram, respectively) was less than that of the
3 days group. With the decrease in the in vivo clearance, the $\mathrm{AUC}_{0-\mathrm{t}}$, $\mathrm{MRT}_{0-\mathrm{t}}$, and $\mathrm{t}_{1 / 2}$ values of the 5 and 7 days groups were higher than those of the 3 days group (Supplemental Table 14). Therefore, the magnitude was gradually decreased as the time interval between doses extended from 3 to 7 days (Table 3). Therefore, repeated injection of PEG-L with a time interval of 3 days could induce the strongest $\mathrm{ABC}$ phenomenon, and the prolongation of the time interval between two injections alleviated the magnitude of the $\mathrm{ABC}$ phenomenon, which is consistent with previous reports ( $\mathrm{Li}$ et al., 2013). Nevertheless, the enhanced accumulations of the second dose in liver and spleen were apparently different among the liposome-treated groups (Fig. 1B). The control rats exhibited very low accumulations of DTX in liver and spleen. Compared with the control group, the lowest concentration of DTX accumulated in the liver of the 3 days group at 12 hours with an obvious ABC effect, while the accumulation of DTX in spleen was far higher than that of the control and other test groups. Interestingly, with the prolonged time interval increased accumulation of the second dose in liver and decreased accumulation in spleen were observed. Inconsistently, compared with the results in Fig. 1B, the relative liver accumulation of DTX in the 3 days group increased by nearly 3 -fold at 4 hours after the second dose (data not shown), while no significant changes were found in the spleen accumulation. Together, these data imply that pretreatment with PEG-L triggers a rapid accumulation of the second dose in liver at the initial time, followed by a remarkable reduction of hepatic DTX in the later hours. We hypothesized that the accelerated metabolism of the drug was caused by P450 enzymes, since it is known that hepatic P450s play an essential role in the process of drug metabolism, which might be induced by repeated injection of PEG-L. It is, therefore, particularly interesting to understand whether the activation of P450s is involved in the $\mathrm{ABC}$ phenomenon.

\section{The Activity and Expression of P450s in Plasma Induced by Single Injection or Repeated Injection of PEG-L}

Determination of $\mathbf{P 4 5 0}$ Activity. In this study, the issue of whether repeated injections of PEG-L will lead to activation of P450s was investigated. A marked difference of pharmacokinetic profiles of several probes was observed between the single and repeated PEG-L injection groups using a probe cocktail assay in rats (Fig. 2; Supplemental Tables 15-17). For midazolam (probe for CYP3A1/2 in Supplemental Table 15), the $\mathrm{CL}_{\mathrm{z}}$ value for the repeated PEG-L injection group $(0.24 \pm 0.07 \mathrm{l} / \mathrm{h}$ per kilogram) was increased significantly $(P<0.01)$ compared with those of the single PEG-L injection group $(0.12 \pm 0.02$ $1 / \mathrm{h}$ per kilogram). Along with the increase in the in vivo clearance, the $\mathrm{AUC}_{0-\mathrm{t}}$ and area under the plasma concentration-time curve from time 0 to infinity values for the repeated PEG-L injection group (4455.52 \pm 1511.12 and $4494.27 \pm 1517.96 \mu \mathrm{g} / \mathrm{l} \cdot \mathrm{h}$, respectively) were significantly decreased $(P<0.01)$ compared with those of the single PEG-L injection group $(7989.68 \pm 1456.23$ and $8266.47 \pm 1490.49 \mu \mathrm{g} / \mathrm{l} \cdot \mathrm{h}$, respectively). In addition, the $t_{1 / 2}$ value for the repeated PEG-L injection group was also decreased compared with that of the single PEG-L injection group $(P<0.05)$. For diclofenac (probe for CYP2C6) in Supplemental Table 16, the $\mathrm{CL}_{\mathrm{z}}$ value for the repeated PEG-L injection group $(1.20 \pm 0.48 \mathrm{l} / \mathrm{h}$ per kilogram) was increased significantly $(P<0.01)$ compared with those of the single PEG-L injection group $(0.26 \pm 0.09 \mathrm{l} / \mathrm{h}$ per kilogram $)$. Similar to midazolam, a corresponding decrease was observed in the $\mathrm{AUC}_{0-\mathrm{t}}$ and area under the plasma concentration-time curve from time 0 to infinity after repeated PEG-L injection $(846.69 \pm 294.10$ and $937.18 \pm 297.09 \mu \mathrm{g} /$ $1 \cdot h$, respectively) compared with those of the single PEG-L injection group $(2734.07 \pm 413.2$ and $4517.64 \pm 2426.29 \mu \mathrm{g} / \mathrm{l} \cdot \mathrm{h}$, respectively) $(P<0.01)$. In addition, the $\mathrm{t}_{1 / 2}, \mathrm{MRT}_{0-\mathrm{t}}$, and mean residence time from time 0 to infinity of the repeated PEG-L injection group also 

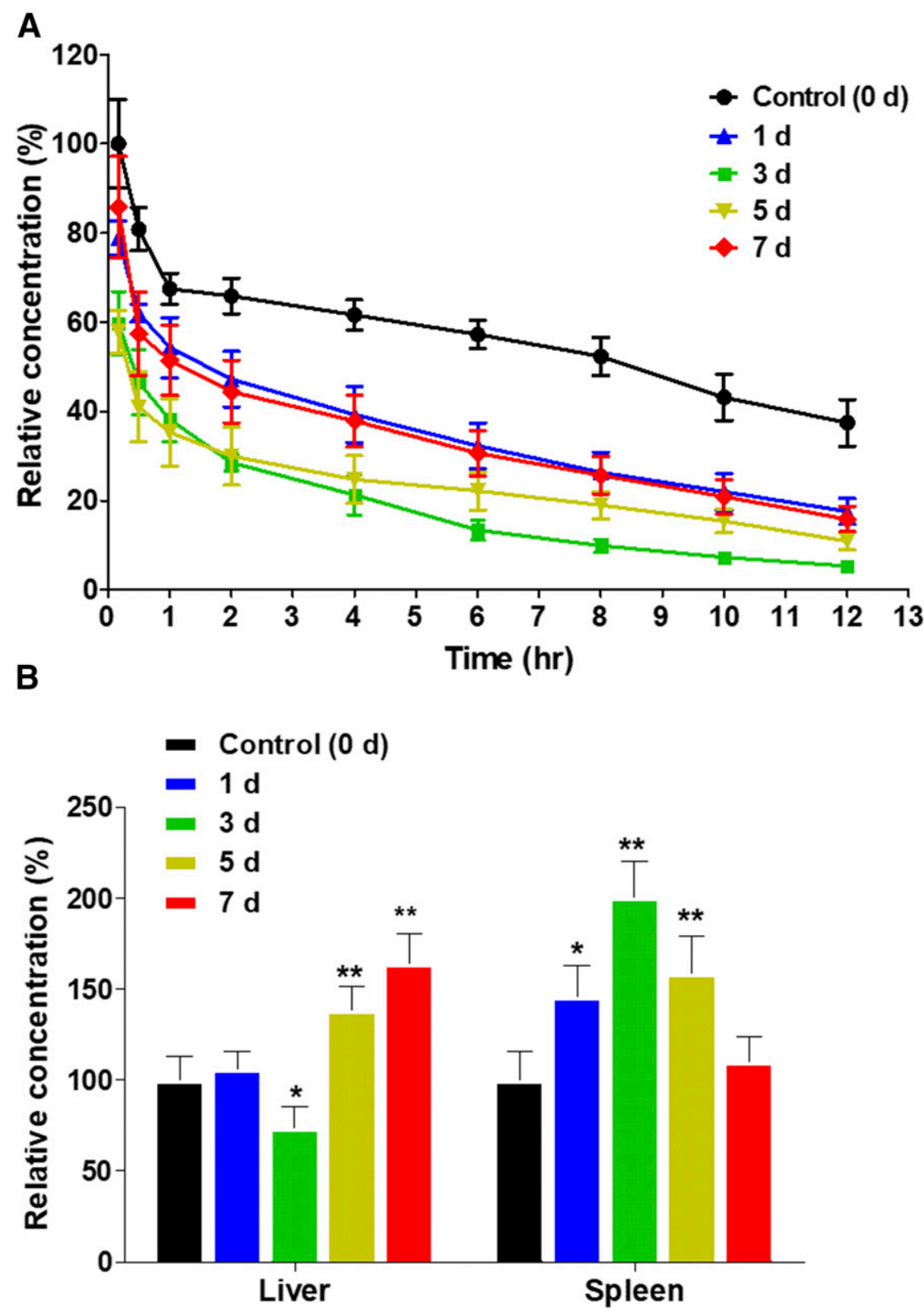

Fig. 1. Effect of the repeated injection time interval on the magnitude of the $\mathrm{ABC}$ phenomenon. (A) The mean concentration-time profile (0-12 hours) of DTX in rats after a single or repeated injection of PEG-L. The relative concentration represents the relative ratio with the initial concentration (at 0.167 hours) of control. (B) Liver and spleen distribution (12 hours) of DTX in rats after the second injection of PEGDTX-L. The relative concentration represents the relative ratio with the liver and spleen concentration of control, respectively. Rats in five groups $(1,3,5$, and 7 days) were injected with two injections of PEG-L at various time intervals $(1,3,5$, and 7 days, respectively). The day 0 group represents a single dose of PEG-L, which is the control for other test groups. $* P<0.05 ; * * P<0.01$ compared with control (day 0$)(n=6$, mean \pm S.D. $)$. decreased compared with those of the single PEG-L injection group $(P<0.01)$. For the CYP1A2 probe phenacetin (Supplemental Table 17), the $\mathrm{CL}_{\mathrm{z}}$ value for the repeated PEG-L injection group $(7.24 \pm 2.87 \mathrm{l} / \mathrm{h}$ per kilogram) was increased significantly $(P<0.01)$ compared with those of the single PEG-L injection group (3.71 \pm $1.38 \mathrm{l} / \mathrm{h}$ per kilogram), and the $\mathrm{AUC}_{0-\mathrm{t}}$ and area under the plasma concentration-time curve from time 0 to infinity values for the repeated PEG-L injection group were decreased significantly $(P<$ $0.01)$. In addition, the $t_{1 / 2}$ of the repeated PEG-L injection group was decreased compared with that of the single PEG-L injection group but did not reach a significant difference. Taken together, these results indicate that the pharmacokinetics of the CYP3A1, CYP2C6, and CYP1A2 probe substrates are altered apparently by repeated injection of PEG-L. No significant difference was detected in the pharmacokinetic characteristics of the CYP2B1 probe bupropion, CYP2C7 probe amodiaquine, CYP2C11 probe omeprazole, and CYP2D2 probe dextromethorphan (data not shown). The pharmacokinetic characteristics of the three probes (midazolam, diclofenac, and phenacetin) were not altered with repeated DTX injections (Supplemental Fig. 9), indicating the P450 response is not due to the burst release of DTX from PEG-L.

Determination of the Expression of P450 mRNA and Protein. Gene transcription and protein expression of CYP3A1, CYP2C6, and CYP1A2 upon repeated injection at different time intervals were evaluated. The expression of hepatic P450 mRNA and protein levels

TABLE 3

Pharmacokinetic parameter ratios for the magnitude of the $\mathrm{ABC}$ phenomenon (mean \pm S.D., $n=6$ )

\begin{tabular}{lccccc}
\hline \multirow{2}{*}{ Parameter } & Control & \multicolumn{4}{c}{ Repeated Injection Group } \\
\cline { 3 - 6 } & & 1 Day & 3 Days & 5 Days & 7 Days \\
\hline $\mathrm{AUC}_{\mathrm{C} / \mathrm{T}}{ }^{a}$ & 1.00 & $1.62 \pm 0.17$ & $3.14 \pm 0.59$ & $2.44 \pm 0.31$ & $1.70 \pm 0.19$ \\
$\mathrm{t}_{1 / 2-\mathrm{C} / \mathrm{T}}$ & 1.00 & $1.38 \pm 0.23$ & $2.54 \pm 0.24$ & $1.62 \pm 0.20$ & $1.50 \pm 0.12$
\end{tabular}

${ }^{a}$ Represents a pharmacokinetic parameter ratio of the control group to the test group. 

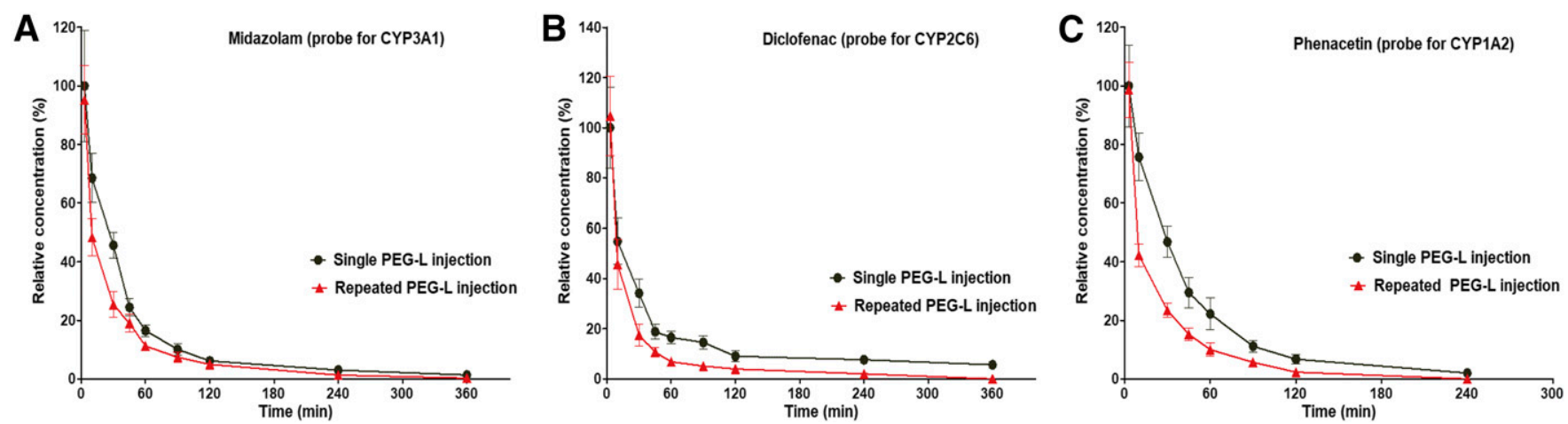

Fig. 2. Effect of a single injection or repeated injection of PEG-L on the pharmacokinetic profiles of midazolam, diclofenac, and phenacetin in rats. (A) The mean concentration-time profile (0-360 minutes) of midazolam (probe for CYP3A1) in rats after the second injection of PEG-L. The relative concentration represents the relative ratio with the initial concentration (at 3 minutes) of a single PEG-L injection equal to 100\%. The impact of repeated PEG-L injections on the plasma concentrations of the CYP2C6 substrate diclofenac and the CYP1A2 substrate phenacetin are presented in (B) and (C), respectively. As described in Table 2, animals in the single PEG-L injection group received a single PEG-L injection followed by intravenous injection of probe cocktail solution after 30 minutes. In the repeated PEG-L injection group, animals received the initial PEG-L injection and a second injection 3 days later, followed by intravenous injection of probe cocktail solution after 30 minutes. Data are presented as the mean \pm S.D. $(n=8)$.

in five groups that received PEG-DTX-L after dosing of PEG-B-L for 0, $1,3,5$, and 7 days were determined. To compare the change in P450 expression profiles clearly, normal control rats received a single injection of equivalent volume PBS. As shown in Fig. 3A, a significant increase in mRNA expression of CYP3A1, CYP2C6, and CYP1A2 was observed in the test groups when compared with control rats. Consistently, the protein expression levels of CYP3A1, CYP2C6, and CYP1A2 were also found to be upregulated in the test groups compared with the control group (Fig. 3B). Concerning CYP3A1 expression, a significant increase in mRNA expression was detected in the 1,3 , and 5 days groups with an apparent increase in protein level in the 3 and 5 days groups. Increased CYP2C6 mRNA and protein levels were found in the 3,5 , and 7 days groups. CYP1A2 mRNA levels were increased in the 3 and 5 days groups and protein levels were elevated in the 3,5 , and 7 days groups. Hence, these data suggest that liver P450 activity differences might be partly, but not absolutely, owing to the differences in their mRNA and protein expression. It is worth mentioning that the mRNA and protein expression of the three P450 isoforms increased in the 1 day group and reached a peak in the 3 days group before gradually decreasing with extended time intervals, which is in accordance with the result of the $\mathrm{ABC}$ magnitude observed in the pharmacokinetic experiments. Meanwhile, the relative concentration of DTX accumulated in liver of the 3 days group fell to the lowest point after the reinjected dose for 12 hours. This suggests P450s might be responsible for the rapid clearance of the second dose in plasma and liver.

\section{Effects of the Presence of P450 Inhibitors on the ABC Phenomenon}

The effects of selective P450 inhibitors on PEG-DTX-L metabolism were investigated by pretreating with vehicle or selective $\mathrm{P} 450$ inhibitors before administration of repeated PEG-L injection with a time interval of 3 days (Fig. 4; Supplemental Table 18). In comparison with the 3 days group (KTZ-), the value of $\mathrm{t}_{1 / 2-\mathrm{C} / \mathrm{T}}$ for the 3 days group (KTZ+) was reduced by 1.88 times, whereas the value of $\mathrm{AUC}_{\mathrm{C} / \mathrm{T}}$ apparently was not decreased. It is worth mentioning that KTZ inhibited the depletion of DTX in the liver. The accumulation of DTX in liver was upregulated dramatically $(P<0.01)$ in the 3 days group for $\mathrm{KTZ}+$ compared with the 3 days group for $\mathrm{KTZ}-$, while comparable results in spleen were found in the two test groups (Fig. 4B). As for CIM, neither the $\mathrm{AUC}_{\mathrm{C} / \mathrm{T}}$ nor $\mathrm{t}_{1 / 2-\mathrm{C} / \mathrm{T}}$ values were significantly decreased in the 3 days group for CIM+ when compared with the 3 days group for CIM- (Fig. 4C; Supplemental Table 18). Likewise, the accumulations of DTX in liver and spleen were not changed with CIM inhibition (Fig. 4D). These results revealed that pretreatment with KTZ attenuated the $\mathrm{ABC}$ magnitude to a small degree.

In addition, the inhibition of KTZ and CIM on hepatic P450 expression was assessed. Pretreatment with KTZ revealed that the mRNA and protein expression of CYP3A1 and CYP1A2 were apparently downregulated compared with those of the 3 days group for KTZ-, while no significant difference was observed for the expression of CYP2C6 (Fig. 5, A and B). The significantly downregulated mRNA and protein expression of CYP2C6 and CYP1A2 were found in the 3 days group for CIM+ in comparison with the 3 days group for CIM-, while no significant difference was observed for the expression of CYP3A1 (Fig. 5, C and D). For the probe substrate DTX, CYP3A (CYP3A4/5 in humans and CYP3A1/2 in rats) may play a more significant role in the substrate, where these enzymes are responsible for the metabolism of the selected probe (Vaclavikova et al., 2004). Therefore, these findings suggest that the significant increase in P450s (especially for CYP3A1) induced by repeated injection of PEG-L is involved in the induction of the ABC phenomenon, whereas CYP2C6 and CYP1A2 play negligible or minor roles.

\section{Discussion}

Despite the superior merits and usefulness of PEGylation in liposomebased therapeutics, increasing evidence suggests that the injection of PEG-L into different species of animals can trigger the $\mathrm{ABC}$ phenomenon, which is an urgent and serious problem to be overcome in the clinical use of liposome-based drug delivery systems (Li et al., 2012, 2013; Suzuki et al., 2014). Previous reports have revealed that the time interval between injections considerably affects the magnitude of the ABC phenomenon (Ishida et al., 2003; Li et al., 2013). Dams et al. (2000) had first shown that preinjection with PEG-L in rats and a rhesus monkey for 7 days induced rapid clearance and enhanced the accumulations of the second injection of PEG-L in liver and spleen, and the extent of this phenomenon was most apparent at a time interval of 6 to 7 days. Subsequently, a series of findings confirmed the optimal time interval for the most effective induction of the $\mathrm{ABC}$ phenomenon is from 3 to 10 days (Li et al., 2012; Saadati et al., 2013). Similar results were obtained for PEG-modified poly-lactic acid nanoparticles (Ishihara et al., 2009). The differences in the types of PEG polymer, physicochemical properties of liposomes (including particle size and zeta potential), animal species, encapsulated drug, as well as dose of PEG-L 

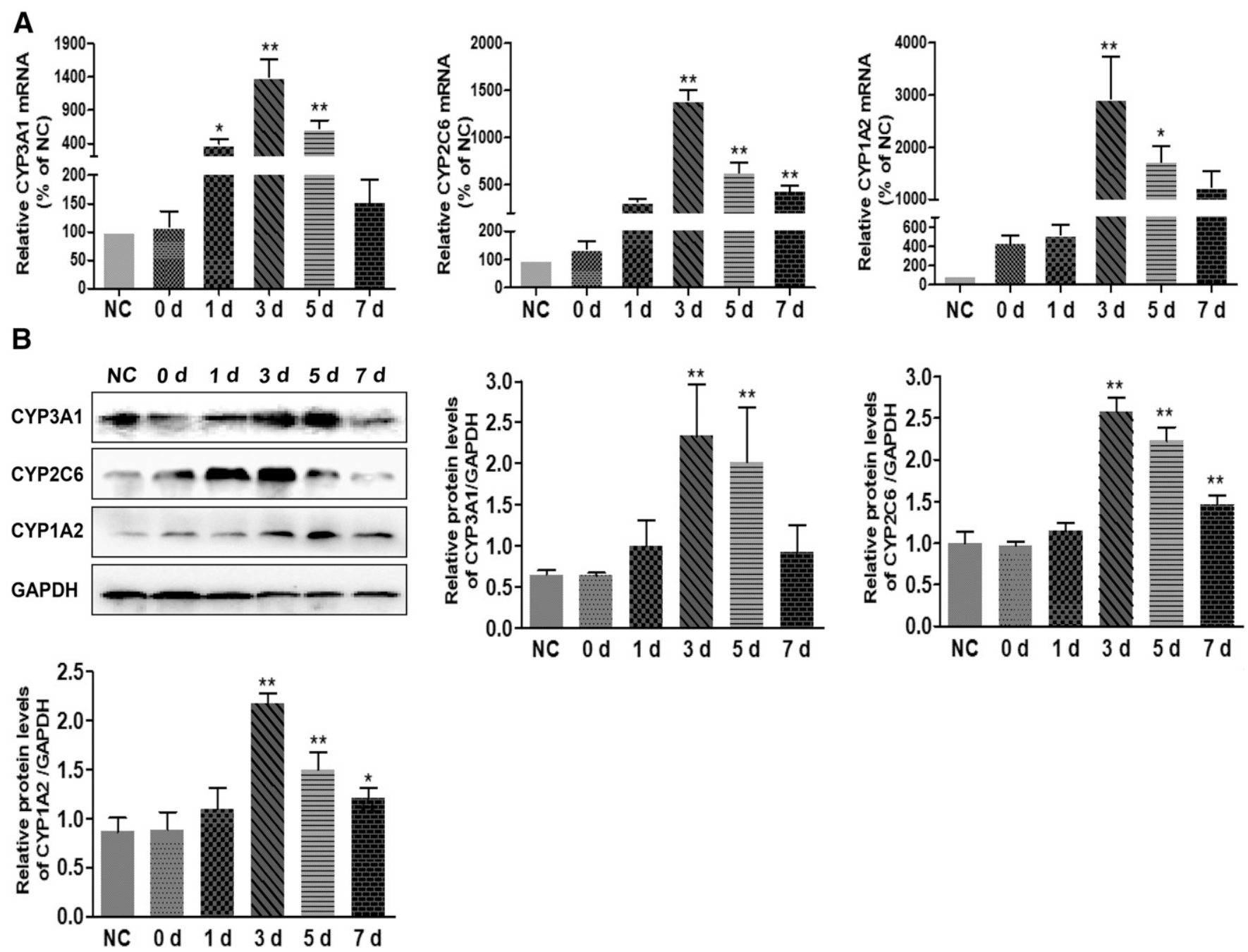

Fig. 3. Effect of single or repeated injection of PEG-L on the mRNA and protein expression of hepatic P450s. The P450 expression profiles were determined after the first injection of PEG-L, followed by a subsequent injection of PEG-DTX-L at different time intervals. The normal control (NC) group was injected with the same volume of PBS as a single injection. After 1, 3,5, and 7 days, the liver was isolated as described in Materials and Methods. (A) Real-time quantitative polymerase chain reaction analysis detected the relative mRNA expression of CYP3A1, CYP2C6, and CYP1A2 in liver. Values were normalized to the respective control groups and fold change was calculated by the $2^{-\Delta \Delta C T}$ method using $\beta$-actin as an endogenous reference gene. (B) Western blot analysis measured the relative protein expression levels of CYP3A1, CYP2C6, and CYP1A2 in liver. Bar graphs show quantitative evaluation of CYP3A1, CYP2C6, and CYP1A2 bands by densitometry from three independent experiments. Each band density was evaluated by ImageJ software and the data were evaluated as percentage compared with glyceraldehyde-3-phosphate dehydrogenase (GAPDH). Day 0 indicates the group received only a single injection of PEG-DTX-L. Days $1,3,5$ and 7 indicate these groups received two injections of PEG-L at different time intervals $(1,3,5$, and 7 days, respectively). Data are presented as the mean \pm S.D. $(n=5$ to 6$)$. $* P<0.05 ; * * P<0.01$ compared with day 0 group.

may affect the time interval for inducing the $\mathrm{ABC}$ phenomenon. For instance, Ishida et al. (2004) reported that the physicochemical properties of the first injected PEG-L (composed of hydrogenated egg phosphatidylcholine, Chol, and $\mathrm{mPEG}_{2000}$-DSPE) strongly affect the extent of the $\mathrm{ABC}$ phenomenon, which was greatly induced with a 10-day interval between injections in their study. Saadati et al. (2013) illustrated that the mass ratio of poly(lactic-co-glycolic acid) (PLGA)-PEG to etoposide was 6:1 and the particle size of etoposide-loaded PLGA-PEG was $173 \mathrm{~nm}$, which were very different from our formulation. In this study, a dose of $0.05 \mu \mathrm{mol} / \mathrm{kg}$ of PEG-B-L was preadministered intravenously to five groups of rats for the first step. The subsequent doses of PEG-DTX-L were injected in each group after $0,1,3,5$, and 7 days. The results showed that a 3-day interval between doses triggered the strongest $\mathrm{ABC}$ phenomenon, and then the magnitude gradually attenuated to a low extent in 7 days (Fig. 1). Furthermore, the prolongation of the time interval between doses could significantly alleviate the $\mathrm{ABC}$ magnitude (Table 3). Herein, the ABC phenomenon might be attenuated and even eliminated when the time interval was long enough.
However, the underlying mechanism of the time interval-dependent $\mathrm{ABC}$ phenomenon has yet to be understood. This phenomenon is widely thought to be caused by increased production of anti-PEG IgM secreted from splenic B cells and subsequent complement activation, leading to the uptake of PEG-L by hepatic and splenic macrophage cells (Shimizu et al., 2015). Nevertheless, it still cannot clearly explain whether the presence of the anti-PEG IgM and complement is totally indispensable (Cui et al., 2008; Zhao et al., 2012), indicating that other factors may also be linked to the $\mathrm{ABC}$ phenomenon.

It is worth mentioning that the induction activity of hepatic P450s is strongly influenced by acute exposure to xenobiotics, which can affect the metabolic process of comedicated drugs by enhancing clearance of the victim drug (Riley and Wilson, 2015). However, whether P450s are involved in the $\mathrm{ABC}$ phenomenon induced by repeated injection of PEG-L has not been verified. To clarify this issue, the relative activity of $\mathrm{P} 450$ isoforms was simultaneously determined by quantitative analysis of classic probe substrates of multiple $\mathrm{P} 450$ s based on a multiple-probe cocktail approach. To avoid potential interactions among the cocktail 
A

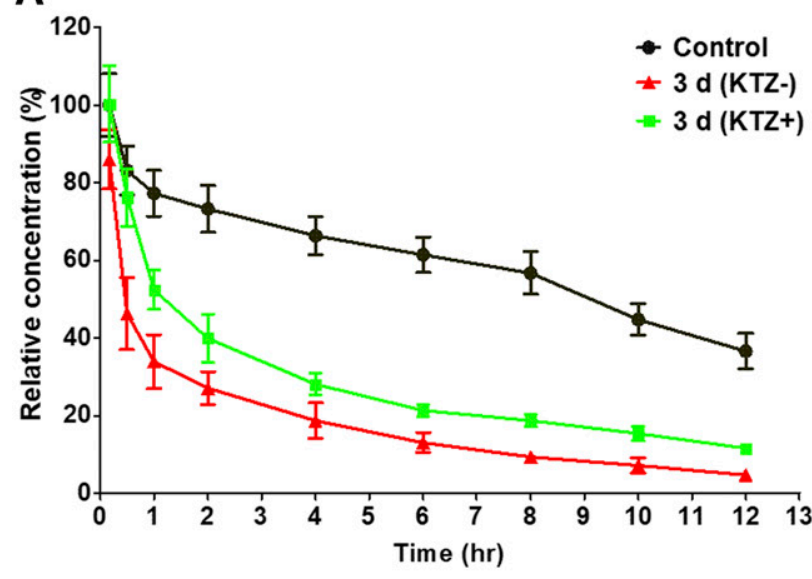

B

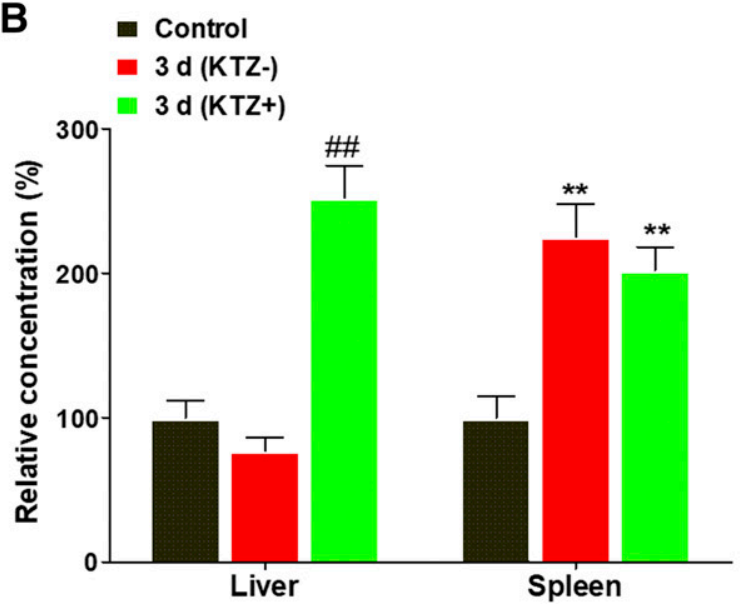

C

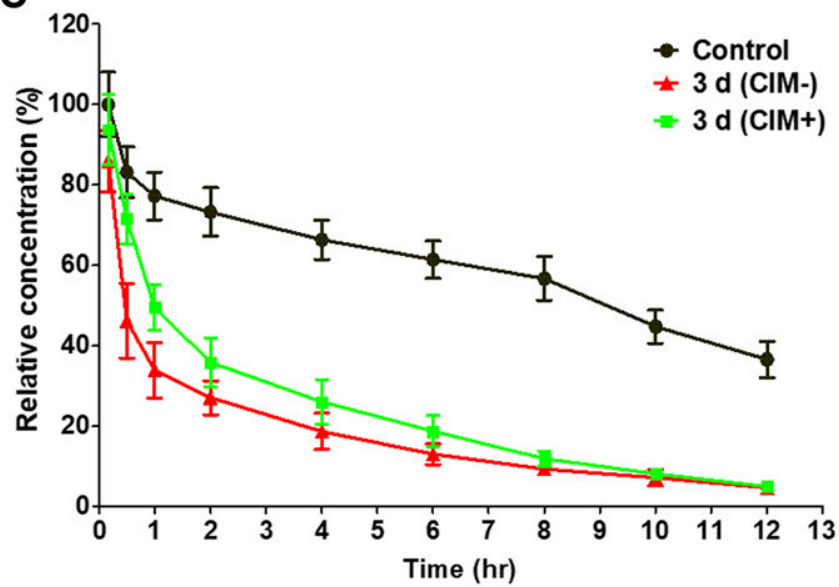

D

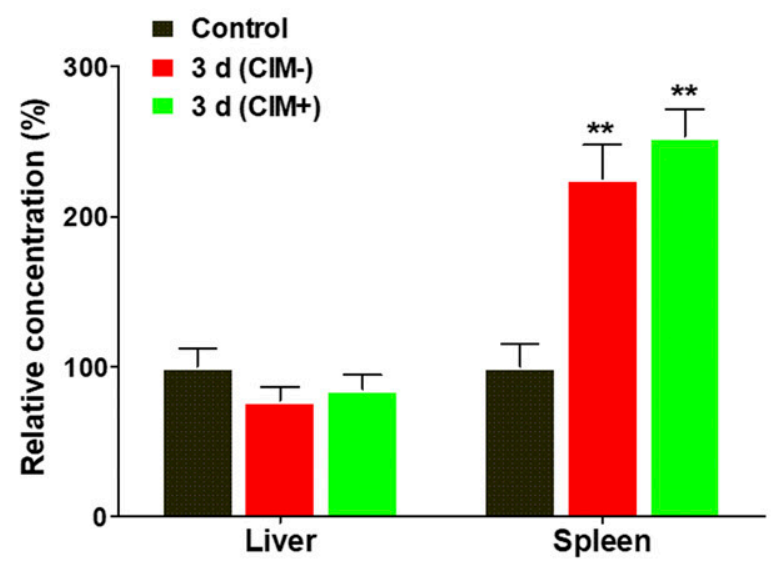

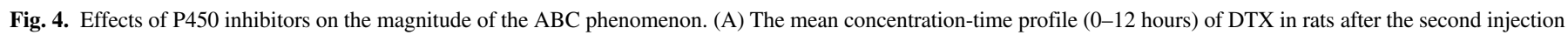

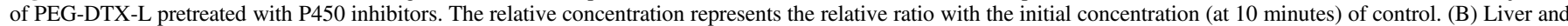

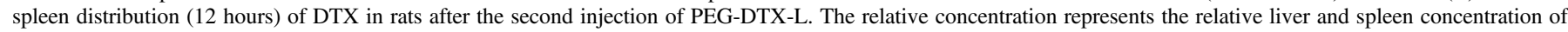

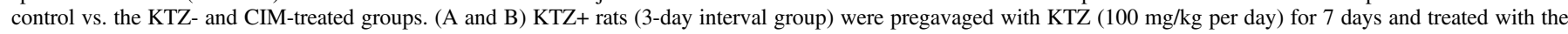

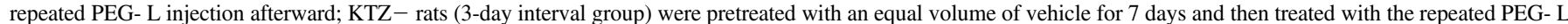

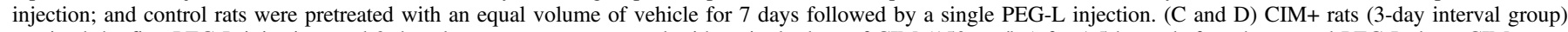

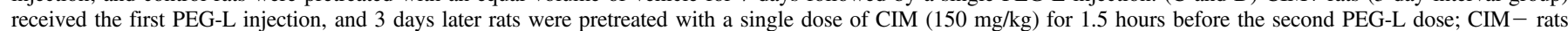

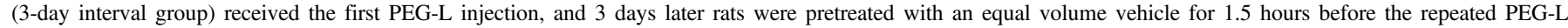

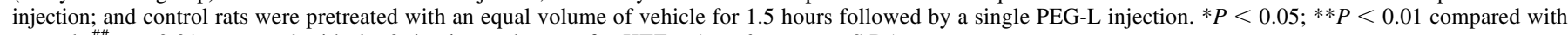
control; ${ }^{\#} P<0.01$ compared with the 3 -day interval group for KTZ $-(n=6$, mean \pm S.D. $)$.

substrates, special care should be taken during the selection of the substrate concentrations in assessing $\mathrm{P} 450$ activity, and reducing the dosage of the substrate is one of the most effective methods. Based on previous studies and our preliminary experiments, the concentrations of probe substrates are $0.4 \mathrm{mg} / \mathrm{kg}$ except for amodiaquine $(1.2 \mathrm{mg} / \mathrm{kg})$ (Han et al., 2014; Sun et al., 2017). Because of the interindividual variation of P450 activity, a sufficient number of subjects is required; therefore, eight rats were used in each group for pharmacokinetic studies to assess the activity of P450s in the current study. Because male rats have 5- to 10-fold higher CYP3A activity than female rats and the level in male rats more closely matches the level in humans, male rats were selected for in vivo experiments (Tomlinson et al., 1997; Bogaards et al., 2000). The activity of CYP3A1, CYP2C6, and CYP1A2 was significantly induced by repeated injection of PEG-L (with a 3-day interval) in terms of altered pharmacokinetic parameters of corresponding probe substrates (Fig. 2; Supplemental Tables 15-17), whereas the activity of CYP2B1, CYP2C7, CYP2C11, and CYP2D2 in the repeated PEG-L injection group was comparable to that of the control group (data no shown). Meanwhile, the highest mRNA and protein expression of CYP3A1, CYP2C6, and CYP1A2 were found in the repeated PEG-L injection group among the aforementioned five groups, which then was gradually reduced with extended time intervals (Fig. 3), which is in accordance with the magnitude of the ABC phenomenon (Table 3). Accordingly, it appears that the increased activity and expression of P450s may have possible involvement in the induction of $\mathrm{ABC}$ phenomenon, which was confirmed by an inhibition study of the $\mathrm{ABC}$ phenomenon in the presence of $\mathrm{P} 450$ inhibitors. From the change in $\mathrm{AUC}_{\mathrm{C} / \mathrm{T}}$ and $\mathrm{t}_{1 / 2-\mathrm{C} / \mathrm{T}}$ values, the $\mathrm{ABC}$ magnitude was slightly suppressed by KTZ pretreatment but not by CIM, and the mRNA and protein of rat CYP3A1 and CYP1A2 were also significantly inhibited (Fig. 5). Notably, when rats were pretreated with KTZ, the relative concentration of DTX in liver was more than 3-fold in rats than without KTZ pretreatment, while the DTX accumulation in spleen was not altered markedly (Fig. 4B). By contrast, the ABC magnitude as well as DTX accumulation in liver and spleen was not affected by the prior administration of CIM (Fig. 4D), indicating a negligible role of CYP2C6 in the ABC phenomenon. These observations may be attributed to the fact that DTX is known to be mainly metabolized by CYP3A1/2, whose activity can be strongly inhibited by KTZ (Kotegawa et al., 2002). Thus, pretreatment with KTZ could suppress the oxidative metabolism of DTX from PEG-DTX-L in plasma and liver, 
A

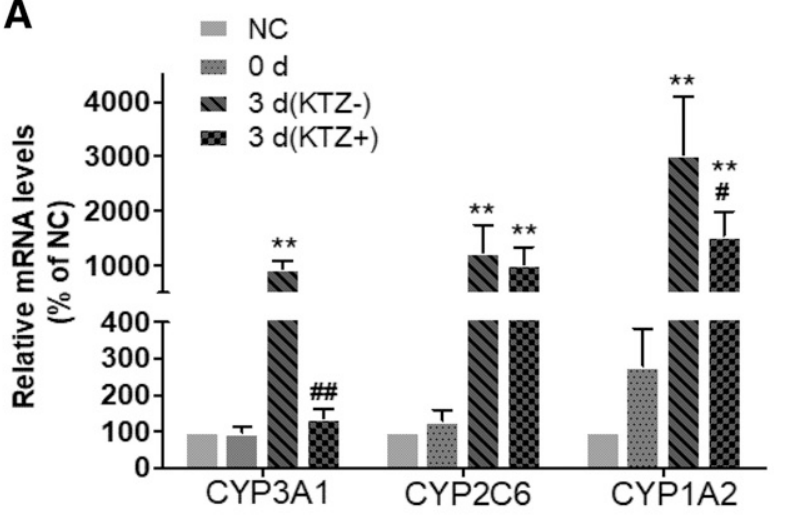

B
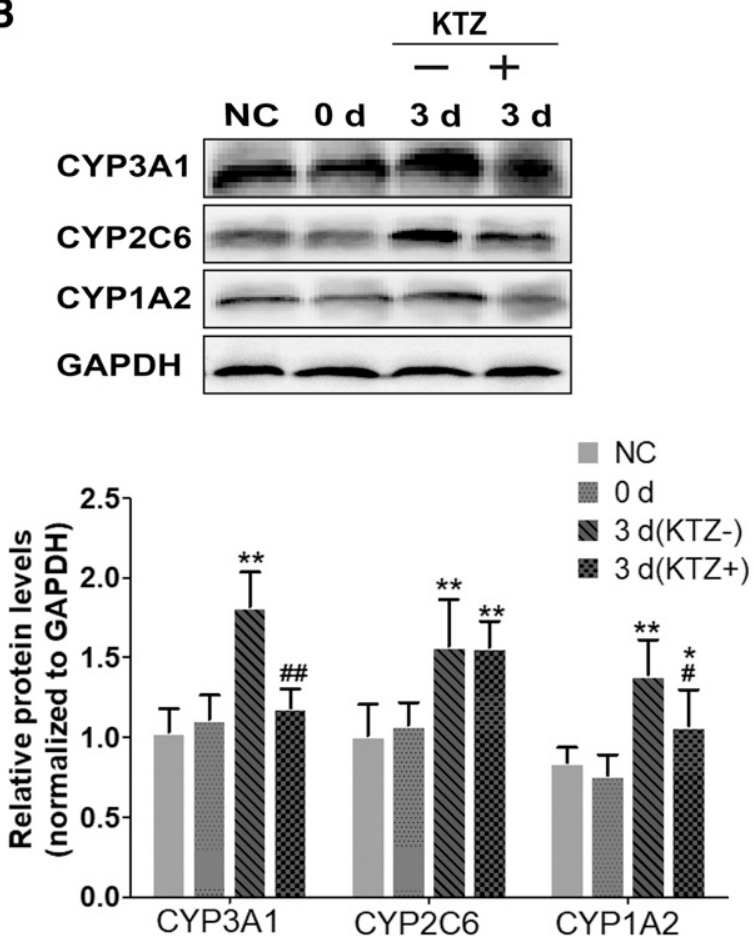

C

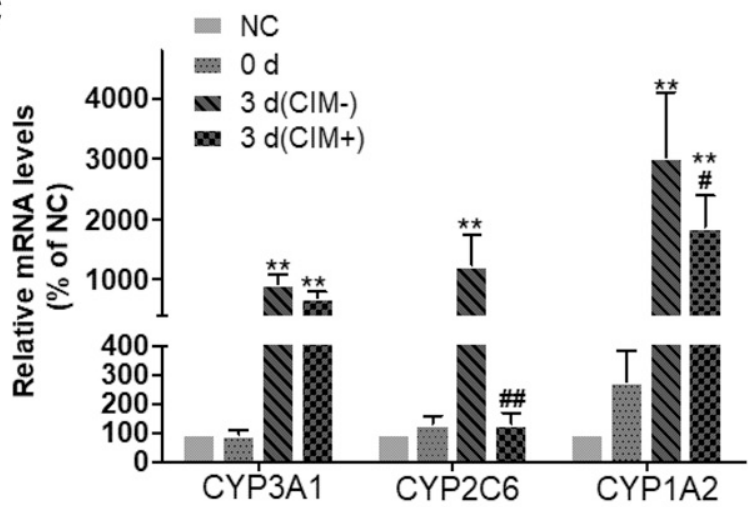

Fig. 5. Effects of the presence of $P 450$ inhibitors on mRNA and protein expression of P450s. By pretreatment with control vehicle or enzyme inhibitors before administration of repeated PEG-L injection with a time interval of 3 days, the respective expression levels of hepatic P450s were determined. (A) Real-time quantitative polymerase chain reaction analysis detected the relative mRNA expression levels of hepatic CYP3A1, CYP2C6, and CYP1A2 in the presence of the CYP3A1 inhibitor KTZ or the CYP2C6 inhibitor CIM (C). Values were normalized to the respective control groups and fold change was calculated by the $2^{-\Delta \Delta C T}$ method using $\beta$-actin as an endogenous reference gene. (B) Western blot analysis measured the relative protein expression levels of hepatic CYP3A1, CYP2C6, and CYP1A2 in the presence of the CYP3A1 inhibitor KTZ or the CYP2C6 inhibitor CIM (D). Bar graphs show quantitative evaluation of CYP3A1, CYP2C6, and CYP1A2 bands by densitometry from three independent experiments. Each band density was evaluated by ImageJ software and the data were evaluated as percentage compared with glyceraldehyde-3-phosphate dehydrogenase (GAPDH). In the 0 day group, animals received a single injection of PEG-L and intravenous injection of probe cocktail solution after 30 minutes. Data are presented as the mean \pm S.D. $(n=5$ to 6$) .{ }^{*} P<0.05 ; * * P<0.01$ compared with the 0 day group; ${ }^{\#} P<0.05 ;{ }^{\# \#} P<0.01$ compared with the 3 days group for KTZ - or the 3 days group for $\mathrm{CIM}-$.

leading to the response of the ABC magnitude of PEG-DTX-L to CYP3A1 inhibition. In this context, CYP3A1 may be involved in the induction of the $\mathrm{ABC}$ phenomenon. Consistent with the work presented in this study, the chemotherapeutic agents topotecan and etoposide are substrates of CYP3A1, and accelerated blood clearance of PEGylated liposomal topotecan and PEGylated PLGA etoposide followed by repeated injections has also been reported (Li et al., 2012; Najar et al., 2011; Skirvin and Lichtman, 2002; Saadati et al., 2013). In contrast, doxorubicin is primarily metabolized to doxorubicinol by cytoplasmic aldo-keto and carbonyl reductases, which may explain why the ABC phenomenon has never been observed in PEGylated liposomal doxorubicin (Robert and Gianni, 1993; Ishida et al., 2006a).
The ABC phenomenon was not abolished with the addition of $\mathrm{P} 450$ selective inhibitors. Apart from P450 induction and anti-PEG IgM, a number of other factors might contribute to the $\mathrm{ABC}$ phenomenon, including the protein corona and lipoprotein receptor. Recently, it has been reported that the protein corona (which mainly consists of complement proteins and apolipoproteins with or without immunoglobulins) formed on polymer nanoparticles including PEG-L plays a predominant role in the clearance of the liposomes from the blood (Pozzi et al., 2014; Papi et al., 2017). In addition, it has been demonstrated that serum albumin and apolipoprotein low-densitylipoprotein receptors also have a positive impact on this phenomenon (Bertrand et al., 2017; Kari et al., 2017). 
The present study has several limitations. First, the P450 activity was investigated by evaluating the original probe substrates rather than their metabolites, and it would be better to illustrate the involvement of specific $\mathrm{P} 450$ isoforms to see a corresponding increase in metabolite generation. Second, although the mRNA and protein of rat CYP1A2 were significantly inhibited by CIM (Fig. 5, C and D), it is yet to be confirmed whether the activity of CYP1A2 was also inhibited; a selective inhibitor of CYP1A2 is, therefore, needed to elucidate it. Surely, more experiments, with stricter parameters, are needed to unveil the underlying mechanisms, such as CYP3A knockout mouse or transgenic mouse models expressing CYP3A, which might give straighter evidence.

Taken together, in light of the data obtained with pharmacokinetics and biodistribution as well as mRNA and protein of rat P450s, this is the first observation indicating that the increased activity and expression of CYP3A1 may be involved in the induction of the ABC phenomenon. However, the relative contribution of $\mathrm{P} 450 \mathrm{~s}$ in the induction process of the $\mathrm{ABC}$ phenomenon remains unknown. Is there a relationship or crosstalk among P450s and other factors in the induction of the ABC phenomenon? Therefore, it is particularly interesting to understand how PEG-L affects the biologic fate of these clinically relevant P450s.

\section{Acknowledgments}

The authors thank all of the members from the Center for Drug Delivery Systems, Shanghai Institute of Materia Medica, Chinese Academy of Sciences, for technical assistance and support in LC-MS/MS.

\section{Authorship Contributions}

Participated in research design: F. Wang, Zhang, Peng, Chen.

Conducted experiments: F. Wang, Wu, $\mathrm{H}$. Wang, Xie.

Performed data analysis: Wu, Zhang, $\mathrm{H}$. Wang, Ye.

Wrote or contributed to the writing of the manuscript: F. Wang, Chen.

\section{References}

Bansal S and DeStefano A (2007) Key elements of bioanalytical method validation for small molecules. AAPS J 9:E109-E114.

Bertrand N, Grenier P, Mahmoudi M, Lima EM, Appel EA, Dormont F, Lim JM, Karnik R, Langer $\mathrm{R}$, and Farokhzad OC (2017) Mechanistic understanding of in vivo protein corona formation on polymeric nanoparticles and impact on pharmacokinetics. Nat Commun 8:777.

Bogaards JJ, Bertrand M, Jackson P, Oudshoorn MJ, Weaver RJ, van Bladeren PJ, and Walther B (2000) Determining the best animal model for human cytochrome P450 activities: a comparison of mouse, rat, rabbit, dog, micropig, monkey and man. Xenobiotica 30:1131-1152.

Burkina V, Rasmussen MK, Pilipenko N, and Zamaratskaia G (2017) Comparison of xenobioticmetabolising human, porcine, rodent, and piscine cytochrome P450. Toxicology 375:10-27.

Cui J, Li C, Wang C, Li Y, Zhang L, Zhang L, and Yang H (2008) Repeated injection of pegylated liposomal antitumour drugs induces the disappearance of the rapid distribution phase. J Pharm Pharmacol 60:1651-1657.

Dams ET, Laverman P, Oyen WJ, Storm G, Scherphof GL, van Der Meer JW, Corstens FH, and Boerman OC (2000) Accelerated blood clearance and altered biodistribution of repeated injections of sterically stabilized liposomes. J Pharmacol Exp Ther 292:1071-1079.

Doak SH and Zaïr ZM (2012) Real-time reverse-transcription polymerase chain reaction: technical considerations for gene expression analysis. Methods Mol Biol 817:251-270.

Gabizon A, Shmeeda H, and Barenholz Y (2003) Pharmacokinetics of pegylated liposomal doxorubicin: review of animal and human studies. Clin Pharmacokinet 42:419-436.

Gai X, Cheng L, Li T, Liu D, Wang Y, Wang T, Pan W, and Yang X (2018) In vitro and in vivo studies on a novel bioadhesive colloidal system: cationic liposomes of ibuprofen. AAPS PharmSciTech 19:700-709.

Guengerich FP (2008) Cytochrome P450 and chemical toxicology. Chem Res Toxicol 21:70-83.

Han YL, Li D, Yang QJ, Zhou ZY, Liu LY, Li B, Lu J, and Guo C (2014) In vitro inhibitory effects of scutellarin on six human/rat cytochrome P450 enzymes and P-glycoprotein. Molecules 19: $5748-5760$.

He RX, Ye X, Li R, Chen W, Ge T, Huang TQ, Nie XJ, Chen HJ, Peng DY, and Chen WD (2017) PEGylated niosomes-mediated drug delivery systems for paeonol: preparation, pharmacokinetics studies and synergistic anti-tumor effects with 5-FU. J Liposome Res 27:161-170.

Huang X, Chen YJ, Peng DY, Li QL, Wang XS, Wang DL, and Chen WD (2013) Solid lipid nanoparticles as delivery systems for gambogenic acid. Colloids Surf B Biointerfaces 102 391-397.

Immordino ML, Dosio F, and Cattel L (2006) Stealth liposomes: review of the basic science, rationale, and clinical applications, existing and potential. Int J Nanomedicine 1:297-315.

Ingelman-Sundberg M (2004) Pharmacogenetics of cytochrome P450 and its applications in drug therapy: the past, present and future. Trends Pharmacol Sci 25:193-200.

Ishida T, Atobe K, Wang X, and Kiwada H (2006a) Accelerated blood clearance of PEGylated liposomes upon repeated injections: effect of doxorubicin-encapsulation and high-dose first injection. J Control Release 115:251-258.
Ishida T, Harada M, Wang XY, Ichihara M, Irimura K, and Kiwada H (2005) Accelerated blood clearance of PEGylated liposomes following preceding liposome injection: effects of lipid dose and PEG surface-density and chain length of the first-dose liposomes. J Control Release 105 : 305-317.

Ishida T, Ichihara M, Wang X, and Kiwada H (2006b) Spleen plays an important role in the induction of accelerated blood clearance of PEGylated liposomes. J Control Release 115: 243-250.

Ishida T, Ichihara M, Wang X, Yamamoto K, Kimura J, Majima E, and Kiwada H (2006c) Injection of PEGylated liposomes in rats elicits PEG-specific IgM, which is responsible for rapid elimination of a second dose of PEGylated liposomes. J Control Release 112: $15-25$.

Ishida T, Ichikawa T, Ichihara M, Sadzuka Y, and Kiwada H (2004) Effect of the physicochemical properties of initially injected liposomes on the clearance of subsequently injected PEGylated liposomes in mice. J Control Release 95:403-412.

Ishida T, Kashima S, and Kiwada H (2008) The contribution of phagocytic activity of liver macrophages to the accelerated blood clearance (ABC) phenomenon of PEGylated liposomes in rats. J Control Release 126:162-165.

Ishida T, Maeda R, Ichihara M, Irimura K, and Kiwada H (2003) Accelerated clearance of PEGylated liposomes in rats after repeated injections. J Control Release 88:35-42.

Ishihara T, Takeda M, Sakamoto H, Kimoto A, Kobayashi C, Takasaki N, Yuki K, Tanaka K, Takenaga M, Igarashi R, et al. (2009) Accelerated blood clearance phenomenon upon repeated injection of PEG-modified PLA-nanoparticles. Pharm Res 26:2270-2279.

Kari OK, Rojalin T, Salmaso S, Barattin M, Jarva H, Meri S, Yliperttula M, Viitala T, and Urtti A (2017) Multi-parametric surface plasmon resonance platform for studying liposome-serum interactions and protein corona formation. Drug Deliv Transl Res 7:228-240.

Koide H, Asai T, Hatanaka K, Akai S, Ishii T, Kenjo E, Ishida T, Kiwada H, Tsukada H, and Oku $\mathrm{N}$ (2010) T cell-independent B cell response is responsible for ABC phenomenon induced by repeated injection of PEGylated liposomes. Int J Pharm 392:218-223.

Koide H, Asai T, Kato H, Ando H, Shiraishi K, Yokoyama M, and Oku N (2012) Size-dependent induction of accelerated blood clearance phenomenon by repeated injections of polymeric micelles. Int J Pharm 432:75-79.

Kotegawa T, Laurijssens BE, Von Moltke LL, Cotreau MM, Perloff MD, Venkatakrishnan K, Warrington JS, Granda BW, Harmatz JS, and Greenblatt DJ (2002) In vitro, pharmacokinetic, and pharmacodynamic interactions of ketoconazole and midazolam in the rat. J Pharmacol Exp Ther 302:1228-1237.

Laginha KM, Verwoert S, Charrois GJ, and Allen TM (2005) Determination of doxorubicin levels in whole tumor and tumor nuclei in murine breast cancer tumors. Clin Cancer Res 11 6944-6949.

Lee B, Ji HK, Lee T, and Liu KH (2015) Simultaneous screening of activities of five cytochrome $\mathrm{P} 450$ and four uridine $5^{\prime}$-diphospho-glucuronosyltransferase enzymes in human liver microsomes using cocktail incubation and liquid chromatography-tandem mass spectrometry. Drug Metab Dispos 43:1137-1146.

Levine M, Law EY, Bandiera SM, Chang TK, and Bellward GD (1998) In vivo cimetidine inhibits hepatic CYP2C6 and CYP2C11 but not CYP1A1 in adult male rats. J Pharmacol Exp Ther 284: 493-499.

Lewis DF, Ioannides C, and Parke DV (1998) Cytochromes P450 and species differences in xenobiotic metabolism and activation of carcinogen. Environ Health Perspect 106: 633-641.

Li C, Cao J, Wang Y, Zhao X, Deng C, Wei N, Yang J, and Cui J (2012) Accelerated blood clearance of pegylated liposomal topotecan: influence of polyethylene glycol grafting density and animal species. J Pharm Sci 101:3864-3876.

Li C, Zhao X, Wang Y, Yang H, Li H, Li H, Tian W, Yang J, and Cui J (2013) Prolongation of time interval between doses could eliminate accelerated blood clearance phenomenon induced by pegylated liposomal topotecan. Int J Pharm 443:17-25.

Lin JH and Lu AY (1998) Inhibition and induction of cytochrome P450 and the clinical implications. Clin Pharmacokinet 35:361-390.

$\mathrm{Lu} \mathrm{C}$ and $\mathrm{Li}$ AP (2001) Species comparison in P450 induction: effects of dexamethasone, omeprazole, and rifampin on P450 isoforms $1 \mathrm{~A}$ and $3 \mathrm{~A}$ in primary cultured hepatocytes from man, Sprague-Dawley rat, minipig, and beagle dog. Chem Biol Interact 134:271-281.

Ma Y, Yang Q, Wang L, Zhou X, Zhao Y, and Deng Y (2012) Repeated injections of PEGylated liposomal topotecan induces accelerated blood clearance phenomenon in rats. Eur J Pharm Sci 45:539-545

Meredith CG, Maldonado AL, and Speeg KV Jr (1985) The effect of ketoconazole on hepatic oxidative drug metabolism in the rat in vivo and in vitro. Drug Metab Dispos 13:156-162.

Mosca P, Bonazzi P, Novelli G, Jezequel AM, and Orlandi F (1985) In vivo and in vitro inhibition of hepatic microsomal drug metabolism by ketoconazole. Br J Exp Pathol 66:737-742.

Najar IA, Sharma SC, Singh GD, Koul S, Gupta PN, Javed S, and Johri RK (2011) Involvement of P-glycoprotein and CYP 3A4 in the enhancement of etoposide bioavailability by a piperine analogue. Chem Biol Interact 190:84-90.

Nishiya Y, Hagihara K, Ito T, Tajima M, Miura S, Kurihara A, Farid NA, and Ikeda T (2009) Mechanism-based inhibition of human cytochrome P450 2B6 by ticlopidine, clopidogrel, and the thiolactone metabolite of prasugrel. Drug Metab Dispos 37:589-593.

Papi M, Caputo D, Palmieri V, Coppola R, Palchetti S, Bugli F, Martini C, Digiacomo L, Pozzi D, and Caracciolo G (2017) Clinically approved PEGylated nanoparticles are covered by a protein corona that boosts the uptake by cancer cells. Nanoscale 9:10327-10334.

Pozzi D, Colapicchioni V, Caracciolo G, Piovesana S, Capriotti AL, Palchetti S, De Grossi S, Riccioli A, Amenitsch H, and Laganà A (2014) Effect of polyethyleneglycol (PEG) chain length on the bio-nano-interactions between PEGylated lipid nanoparticles and biological fluids: from nanostructure to uptake in cancer cells. Nanoscale 6:2782-2792.

Riley RJ and Wilson CE (2015) Cytochrome P450 time-dependent inhibition and induction: advances in assays, risk analysis and modelling. Expert Opin Drug Metab Toxicol 11: 557-572.

Rio D, Ares M, Hannon G, and Nilsen T (2010) Purification of RNA using TRIzol (TRI reagent). Cold Spring Harb Protoc 2010:pdb.prot5439.

Robert J and Gianni L (1993) Pharmacokinetics and metabolism of anthracyclines. Cancer Surv 17: 219-252.

Roth R, Madhani HD, and Garcia JF (2018) Total RNA isolation and quantification of specific RNAs in fission yeast. Methods Mol Biol 1721:63-72.

Saadati R, Dadashzadeh S, Abbasian Z, and Soleimanjahi H (2013) Accelerated blood clearance of PEGylated PLGA nanoparticles following repeated injections: effects of polymer dose, PEG coating, and encapsulated anticancer drug. Pharm Res 30:985-995. 
Shimizu T, Mima Y, Hashimoto Y, Ukawa M, Ando H, Kiwada H, and Ishida T (2015) Anti-PEG IgM and complement system are required for the association of second doses of PEGylated liposomes with splenic marginal zone B cells. Immunobiology 220:1151-1160.

Skirvin JA and Lichtman SM (2002) Pharmacokinetic considerations of oral chemotherapy in elderly patients with cancer. Drugs Aging 19:25-42.

Suk JS, Xu Q, Kim N, Hanes J, and Ensign LM (2016) PEGylation as a strategy for improving nanoparticle-based drug and gene delivery. Adv Drug Deliv Rev 99:28-51.

Sun J, Lu Y, Li Y, Pan J, Liu C, Gong Z, Huang J, Zheng J, Zheng L, Li Y, et al. (2017) Influence of shenxiong glucose injection on the activities of six CYP isozymes and metabolism of warfarin in rats assessed using probe cocktail and pharmacokinetic approaches. Molecules 22:1994.

Suzuki T, Ichihara M, Hyodo K, Yamamoto E, Ishida T, Kiwada H, Kikuchi H, and Ishihara H (2014) Influence of dose and animal species on accelerated blood clearance of PEGylated liposomal doxorubicin. Int J Pharm 476:205-212.

Tomlinson ES, Maggs JL, Park BK, and Back DJ (1997) Dexamethasone metabolism in vitro: species differences. J Steroid Biochem Mol Biol 62:345-352.

Tracy TS, Chaudhry AS, Prasad B, Thummel KE, Schuetz EG, Zhong XB, Tien YC, Jeong H, Pan $\mathrm{X}$, Shireman LM, et al. (2016) Interindividual variability in cytochrome P450-mediated drug metabolism. Drug Metab Dispos 44:343-351.

Turpeinen M, Hofmann U, Klein K, Mürdter T, Schwab M, and Zanger UM (2009) A predominate role of CYP1A2 for the metabolism of nabumetone to the active metabolite, 6-methoxy-2naphthylacetic acid, in human liver microsomes. Drug Metab Dispos 37:1017-1024.

Uoto K, Mitsui I, Terasawa H, and Soga T (1997) First synthesis and cytotoxic activity of novel docetaxel analogs modified at the C18-position. Bioorg Med Chem Lett 7:2991-2996.

Vaclavikova R, Soucek P, Svobodova L, Anzenbacher P, Simek P, Guengerich FP, and Gut (2004) Different in vitro metabolism of paclitaxel and docetaxel in humans, rats, pigs, and minipigs. Drug Metab Dispos 32:666-674.
Walsky RL and Obach RS (2004) Validated assays for human cytochrome P450 activities. Drug Metab Dispos 32:647-660.

Wang X, Han A, Wen C, Chen M, Chen X, Yang X, Ma J, and Lin G (2013) The effects of $\mathrm{H}_{2} \mathrm{~S}$ on the activities of CYP2B6, CYP2D6, CYP3A4, CYP2C19 and CYP2C9 in vivo in rat. Int J Mol Sci 14:24055-24063.

Xu H, Ye F, Hu M, Yin P, Zhang W, Li Y, Yu X, and Deng Y (2015) Influence of phospholipid types and animal models on the accelerated blood clearance phenomenon of PEGylated liposomes upon repeated injection. Drug Deliv 22:598-607.

Yang L, Yan C, Zhang F, Jiang B, Gao S, Liang Y, Huang L, and Chen W (2018) Effects of ketoconazole on cyclophosphamide metabolism: evaluation of CYP3A4 inhibition effect using the in vitro and in vivo models. Exp Anim 67:71-82.

Yang Q, Ma Y, Zhao Y, She Z, Wang L, Li J, Wang C, and Deng Y (2013) Accelerated drug release and clearance of PEGylated epirubicin liposomes following repeated injections: a new challenge for sequential low-dose chemotherapy. Int J Nanomedicine 8:1257-1268.

Zhao Y, Wang C, Wang L, Yang Q, Tang W, She Z, and Deng Y (2012) A frustrating problem: accelerated blood clearance of PEGylated solid lipid nanoparticles following subcutaneous injection in rats. Eur J Pharm Biopharm 81:506-513.

Address correspondence to: Weidong Chen, School of Pharmaceutical Sciences, Anhui University of Chinese Medicine, Qianjiang 1, Hefei 230012, Anhui, China. E-mail: wdchen@ahtcm.edu.cn; or Daiyin Peng, School of Pharmaceutical Sciences, Anhui University of Chinese Medicine, Qianjiang 1, Hefei 230012, Anhui, China. E-mail: pengdy@ahtcm.edu.cn 